\title{
Aluminium Complexes: Next-Generation Catalysts in Selective Hydroboration
}

\begin{abstract}
Amrita Das, ${ }^{* a}$ Supriya Rej ${ }^{* b}$ and Tarun K. Panda *c
Organoboranes obtained from hydroboration reactions are one of the important classes of compounds that could be used to provide valuable synthons for follow-up transformations such as various functional group incorporation or C-C bond forming reactions. For decades, various transition metals were utilised as catalysts in such transformations. Recently earth-abundant and less toxic main group metals are revived their importance in hydroboration chemistry, among which the suitable candidates are aluminium complexes as catalysts. In this regard, the development of aluminium complexes to achieve more robust catalytic systems with greater efficacy is appreciable.
\end{abstract}

\section{Introduction}

To push scientific developments towards more sustainable and greener paths, researchers are seeking out less toxic and environmentally benign synthetic transformations. Given these objectives, rapid development and use of synthetic intermediates has occurred in parallel. Focussing on the chemistry of organic intermediates, organoboranes constitute a substantial part of this field of research. Amongst various methods used to synthetically prepare useful organoboranes, one of the most fundamental is the hydroboration of any unsaturated $\mathrm{C}-\mathrm{X}$ bond. ${ }^{1-12}$ The first example of hydroboration was the pioneering work by Brown et al. where a $\mathrm{H}-\mathrm{B}$ bond was added to an alkene in anti-Markovnikov fashion (Scheme 1a). ${ }^{13-}$ 15 The most straightforward hydroboration reagent involves the use of diborane $\left(\left(\mathrm{BH}_{3}\right)_{2}\right)$ or adduct formed with THF $\left(\mathrm{BH}_{3} \cdot \mathrm{THF}\right)$ or alkyl borane (Scheme $1 \mathrm{~b}$ ). ${ }^{15}$ All these reagents are very active, which make them pyrophoric and difficult to handle, and due to elevated activity, uncontrolled reactions may occur. Compared to these traditional and highly reactive boron sources, milder, less toxic and easier to handle boranes, such as catecholborane (HBcat), pinacolborane (HBpin) and others were found to be suitable hydroboration reagents (Scheme 1b). ${ }^{16}$ These discoveries showed that while on the one hand, more controlled selective reactions could take place using the milder alternatives, on the other, their use required high reaction temperatures to compensate for their decreased reactivity.

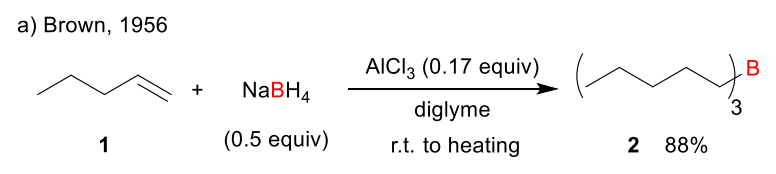

b) Scope of hydroboration reagents

$$
\begin{gathered}
\mathrm{NaBH}_{4}+\mathrm{AlCl}_{3} \\
\mathrm{NaBH}_{4}+\mathrm{BF}_{3} \cdot \mathrm{Et}_{2} \mathrm{O} \\
\left(\mathrm{BH}_{3}\right)_{2} \\
\mathrm{BH}_{3} \cdot \mathrm{THF} \\
\mathrm{BH}_{3} \cdot \mathrm{SMe}_{2}
\end{gathered}
$$

$\mathrm{BR}_{2} \mathrm{H}$ and $\mathrm{MBR}_{3} \mathrm{H}$

$$
\mathrm{R}=\text { alkyl/aryl }
$$

\begin{tabular}{l|c}
$\checkmark$ Highly reactive & Mild, controlled reaction \\
$\checkmark$ Proceeds at $0{ }^{\circ} \mathrm{C}$ to room temp. & Demands catalytic condition \\
$\mathrm{X}$ Pyrophoric, hazardous & $\mathrm{X}$ Requires elevated temp. \\
$\mathrm{X}$ Uncontrolled reaction & without using catalyst \\
\hline
\end{tabular}

c) Nöth, 1985

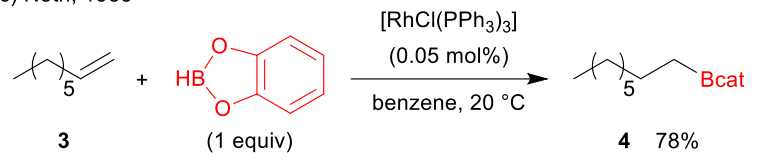

d) Hreczycho, 2018

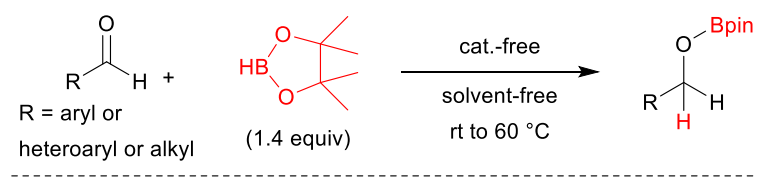

e) Thomas, 2020
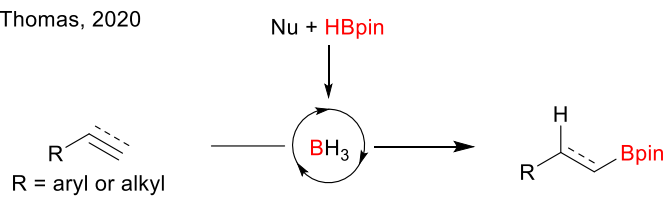

Scheme 1 a) First report of hydroboration reaction. b) Scope of various hydroboration reagents. c) First report of catalytic version of hydroboration reaction. d) Catalyst free and solvent free hydroboration of aldehydes. e) Role of hidden boron catalysis in hydroboration of alkynes and alkenes.

Several research areas in chemistry are evolving every day owing to catalysis. Hence, by maintaining its true importance and essence in organic chemistry, several transition metal complexes efficiently engaged in catalytic hydroboration 
reaction with mild borane reagents. ${ }^{17-19}$ An early discovery in this field involved the successful employment of the Rh-catalyst (well known as Wilkinson's catalyst) in the hydroboration of alkenes with HBpin, as reported by Nöth et al. (Scheme 1c). ${ }^{20}$ The corresponding hydroboration product was formed selectively, following anti-Markovnikov fashion. In the early 90s,' some catalyst-free reports were also published by Knochel ${ }^{21}$ and Piers. ${ }^{22,23}$ The reaction of pinacolborane (HBpin) and highly electrophilic borane $\left(\mathrm{HB}\left(\mathrm{C}_{6} \mathrm{~F}_{5}\right)_{2}\right)$ with alkenes and alkynes resulted in the corresponding hydroboration products without any catalyst. However, these catalyst-free processes also suffered from limitations as uncontrolled reactions took place to produce a mixture of mono- and di-hydroboration products from alkynes. The issue of regioselectivity was another concern for internal alkynes. Recently, Hreczycho et al. reported efficient and mild hydroboration of aldehydes without the need for any catalyst under neat conditions (Scheme 1d). ${ }^{24}$ The neat condition was crucial to furnish a high yield of the desired product as the use of solvents sharply decreased the efficiency. Recently, Thomas et al. discussed the concept of hidden boron catalysis in the hydroboration of alkynes and alkenes. ${ }^{12,25}$ Nucleophiles such as $\mathrm{NaO}{ }^{t} \mathrm{Bu},{ }^{n} \mathrm{Buli},{ }^{n} \mathrm{Bu}_{2} \mathrm{Mg}, \mathrm{Na}\left[\mathrm{N}\left(\mathrm{SiMe}_{3}\right)_{2}\right]$ promoted the hydroboration reaction by the generation of $\mathrm{BH}_{3}$ and borohydride species from the decomposition of stoichiometrically used HBpin (Scheme 1e). ${ }^{25}$ These nucleophiles only promoted the reaction by delivering $\mathrm{BH}_{3}$ which was actual catalytic species and termed as a hidden hydroboration catalyst. The corresponding product formed is the most well-discussed substrate for Suzuki-Miyaura coupling ${ }^{26-28}$ and Chan-Lam coupling. ${ }^{29,30}$ The evaluation of various transition metals as catalysts for hydroboration reactions was well developed. In comparison, the chemistry of main group metals and lanthanides in the insertion of $\mathrm{H}-\mathrm{B}$ bonds into unsaturated moieties has been less explored. However, there was no significant advancement of research into aluminium of group 13 metals, which was thought to be a suitable alternative to transition metal catalysts, until the recent ground-breaking discovery by Yang, Parameswaran and Roesky. ${ }^{31}$ Subsequently, there has been notable progress in this field of research. Given this background, we believe this is a good time to give researchers an informative overview of aluminium-catalysed hydroboration. In this Frontiers review, our main aim is to discuss the advantages of aluminium complexes and their key appearances as selective catalysts in hydroboration chemistry.

\section{Why aluminium and how it stole the show}

Aluminium is one of the most abundant metals found in the earth's crust. It is also useful from an environmental and economic point of view due to its low toxicity and costeffectiveness. ${ }^{32}$ Its usefulness became apparent from one of organic chemistry's earliest and most ground-breaking discoveries, Friedel-Craft's acylation. ${ }^{33-35}$ Over the years, aluminium lost its way as a catalyst due to the frequent appearance and success of transition metals in the catalysis of various organic reactions. As discussed earlier, due to the need for highly reactive reagents, very reactive $\mathrm{NaBH}_{4}$ or direct addition of $\mathrm{BH}_{3}$ is traditionally used as a hydroboration reagent. While $\mathrm{LiAlH}_{4}$ could also be used in similar transformations, i.e., hydroalumination followed by trans-metalation to grow hydroboranes, these stoichiometric metal hydrides are toxic, generating large quantities of metal waste and are dangerous to use in bulk scale reactions. For decades, various transition metals were utilised as catalysts in such transformations and were undoubtedly appreciated for their contribution. Nowadays, however, in every area of research, there is a search for green alternatives. For the sake of developing a sustainable world in general, recently earth-abundant and less toxic metals are reviving their importance in hydroboration and borylation chemistry, among which the suitable candidates are aluminium complexes as catalysts. ${ }^{36-38}$ The reactivity of aluminium complexes can be controlled by suitably tuning their ligands, and selective reduction of various functionalities via hydroboration can be achieved (Scheme 2A). Moreover, the amount of catalyst can be decreased to as low as $0.5-1 \mathrm{~mol} \%$ in several Al-catalysed hydroboration reactions.

A thorough examination of published literature indicates that no summarised review is available on the topic of selective hydroboration reactions catalysed by aluminium complexes, despite ongoing interest in recent years. In 2017 and 2018, a few reviews were published on the application of soluble aluminium hydrides as catalysts in deprotonation, insertion, activation reactions, and reduction of functional groups. $32,39,40$ However, these reviews were not up to date and covered only a limited area. In view of recent progress, we believe that the evolution of aluminium complexes as hydroboration catalysts is a much-discussed field that needs to be reviewed. This review will mainly focus on how aluminium complexes were developed to catalyse controlled and selective hydroboration of various functional groups such as carbonyls, imines, alkenes, alkynes and nitriles (Scheme 2B).

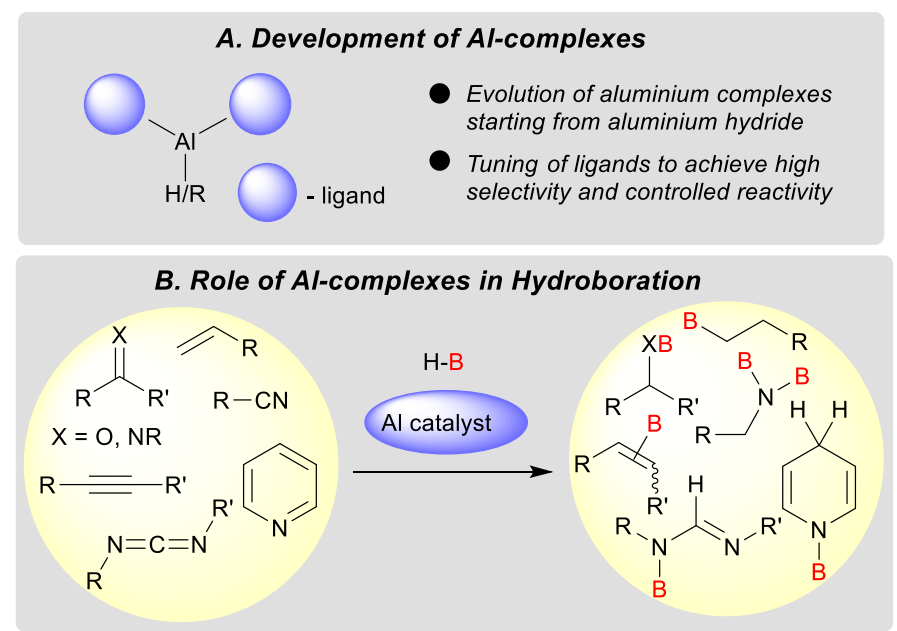

Scheme 2 Development of Al-complexes and their application in the hydroboration of various unsaturated compounds. 


\section{Al-complex catalysed hydroboration}

\subsection{Hydroboration of carbonyl compounds}

In 1979, Noyori et al. reported the BINOL-derived aluminium hydride (BINAL-H)-promoted enantioselective reduction of ketones with $100 \%$ enantioselectivity. ${ }^{41-43}$ After Noyori's seminal work, in 2000, a catalytic version of hydroboration of prochiral ketones was reported by Woodward (Scheme 3). ${ }^{44}$ To achieve an improved catalytic system compared to BINAL-H and avoid the removal of the chiral auxiliary from the metal centre, they hypothesised that the concept of HSAB principle might work well. As the resulting chiral sec-alkoxide contains a hard oxygen donor, HBcat was used as a hard hydride source. Along with chiral BINOL (1,1'-(bi-2-naphthol, BINOLH $\left.{ }_{2}\right)$, other softer analogues of BINOL, such as 2-hydroxy-2'-mercapto-1,1'binaphthyl $\left(\mathrm{MTBH}_{2}\right)$ and 2,2'-dimercapto-1,1'-binaphthyl $\left(\mathrm{DTBH}_{2}\right)$ were combined with $\mathrm{LiMH}_{4}(\mathrm{M}=\mathrm{Al}, \mathrm{Ga})$ to generate the chiral catalyst in situ (Scheme 3). Although the reaction achieved high yields from both metal sources, in the case of the aluminium complex, the enantioselectivity dropped to as low as $1 \%$. On the other hand, softer gallium Lewis acid in combination with a soft thiolate-derived ligand $\mathrm{MTBH}_{2}$ yielded high enantioselectivity.

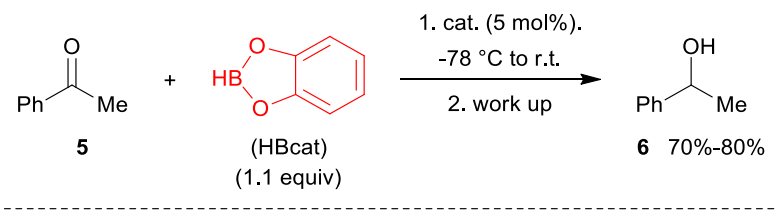

$\mathrm{BINOLH}_{2} \mathrm{~A}=\mathrm{B}=\mathrm{OH}$
$\mathrm{MTBH}_{2} \mathrm{~A}=\mathrm{SH}, \mathrm{B}=\mathrm{OH}$

Scheme 3 Enantioselective hydroboration followed by hydrolysis of ketones.
Yang/Parameswaran/Roesky, 2015

a)

$$
\mathrm{R}_{\mathrm{R}^{2}}^{\text {ㄴ }}
$$<smiles>CCCCCCCCCCCCCCC</smiles>

${ }_{R_{H}^{1}}^{O^{-B p i n}}$

$\mathrm{R}^{1}=$ aryl or alkyl $\mathrm{R}^{2}=$ alkyl or $\mathrm{H}$ (HBpin) (1 equiv)

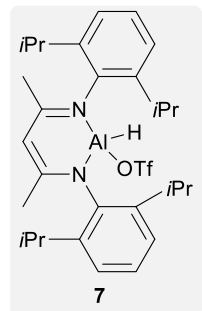

11 examples $51 \%-99 \%$

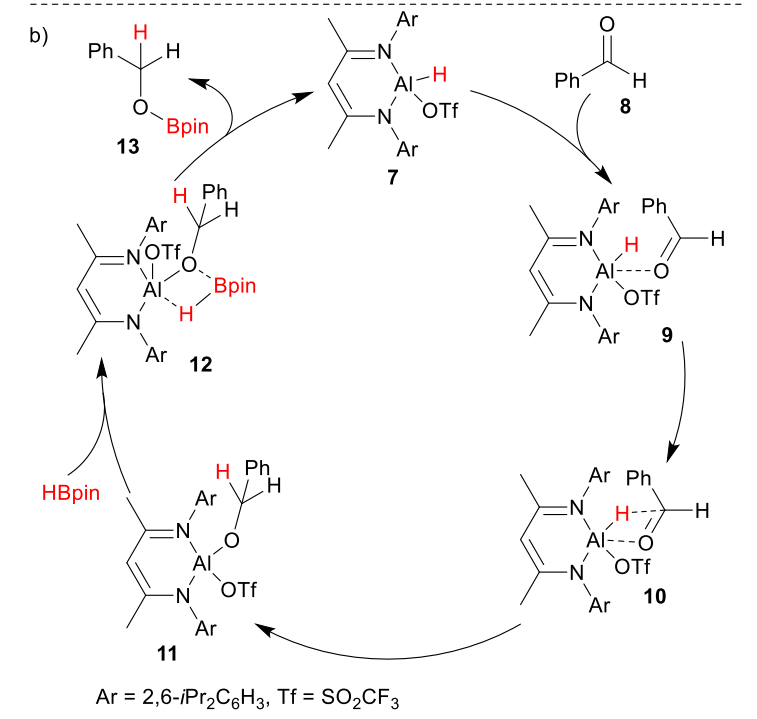

Scheme $4 \beta$-diketimine ligated Al-complex catalysed hydroboration of carbonyl compounds.

In 2015, Yang, Parameswaran and Roesky et al. reported, for the first time, organoaluminium hydride catalysed hydroboration of carbonyl compounds. ${ }^{31}$ They showed that 1,3-diketimine ligated Al-complex (7) could efficiently catalyse the hydroboration of several aldehydes and ketones under mild conditions, with catalyst loading being as low as $1 \mathrm{~mol} \%$ (Scheme 4a). The mechanism they proposed suggested an initial hydride transfer from catalyst 7 to benzaldehyde (Scheme $4 \mathrm{~b}$ ). The resulting $\mathrm{Al}-\mathrm{O}$ bond of the Al-alkoxide complex (11) participates in $\sigma$-bond metathesis with the $\mathrm{B}-\mathrm{H}$ bond of HBpin to yield the desired product (13). The theoretical calculation also supported the insertion/ $\sigma$ bond metathesis pathway as the mechanism for the hydroboration reaction.

After the pioneering work by Roesky et al., several research groups reported hydroboration of aldehydes and ketones under mild conditions with minimal catalyst loading (Scheme 5a). During this research, new developments regarding the structure of aluminium complexes were seen, whether it was tuning ligands or the nature of the metal itself. $N$-donor-supported aluminium monohydride complex $\mathbf{1 4}$ was demonstrated as an efficient catalyst in the hydroboration of functionally diverse carbonyl compounds by Nembenna et al. (Scheme 5a).45 Moreover, this suitably designed catalyst (14) could facilitate 
the chemo-selective hydroboration of aldehydes over ketones in intermolecular reactions at room temperature. $\mathrm{N}$ heterocyclic imines (NHIs) stabilised aluminium hydride triflates (15 and 16) were studied as effective catalysts in the hydroboration of carbonyl compounds by Inoue et al. ${ }^{46}$ The synthesis of various bimetallic lithium aluminates (such as 17) were studied by Okuda, Mulvey and co-workers (Scheme 5a). ${ }^{47-}$ ${ }^{49} \mathrm{~A}$ series of heteroleptic mono-amidomonohydrido, as well as diamidodihydrido, aluminate complexes were synthesised and their catalytic activity was implemented in the hydroboration of several aldehydes and ketones. Very recently, Nembenna et al. showcased the synthesis of conjugated bis-guanidine (CBG) ligated dialkyl aluminium complex (18) and its application in the hydroboration of a wide range of aldehydes and ketones with HBpin (Scheme 5a). ${ }^{50}$ The reactive catalytic species aluminium alkyl-hydride was generated from the reaction between aluminium dialkyls and HBpin in the first step of the reaction mechanism. An interesting study of catalytic activity between the bimetallic lithium aluminates $(\mathbf{2 1}, \mathbf{2 3}, \mathbf{2 5})$ and the corresponding neutral aluminium complexes $(\mathbf{2 2}, \mathbf{2 4}, \mathbf{2 6})$ was investigated by McLellan and Mulvey et al. (Scheme 5b). ${ }^{49}$ In general, it was observed that the cooperative effect of Li-Al in the aluminate complexes exhibited superior reactivity compared to the neutral Al-complexes in most cases. Complex 22, though lacking the $\mathrm{Al}-\mathrm{H}$ bond, could still be used as a source of masked hydride through a $\beta-\mathrm{H}$ transfer process.

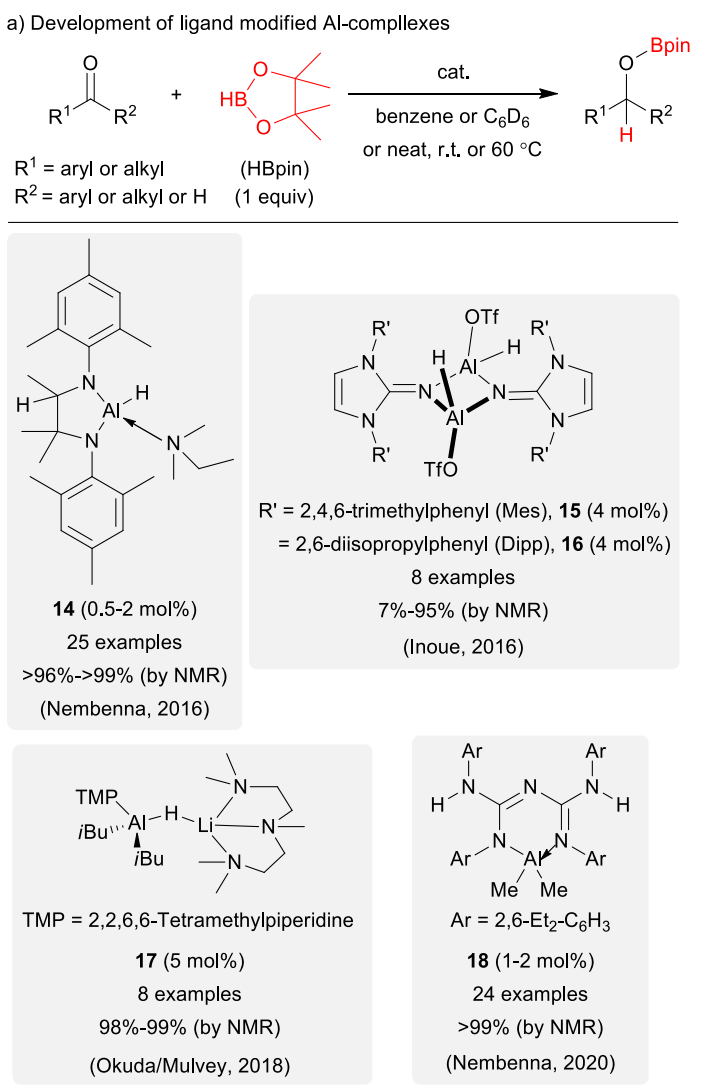

b) Comparison of Al-ate vs neutral Al-complexes (McLellan/Mulvey, 2018)
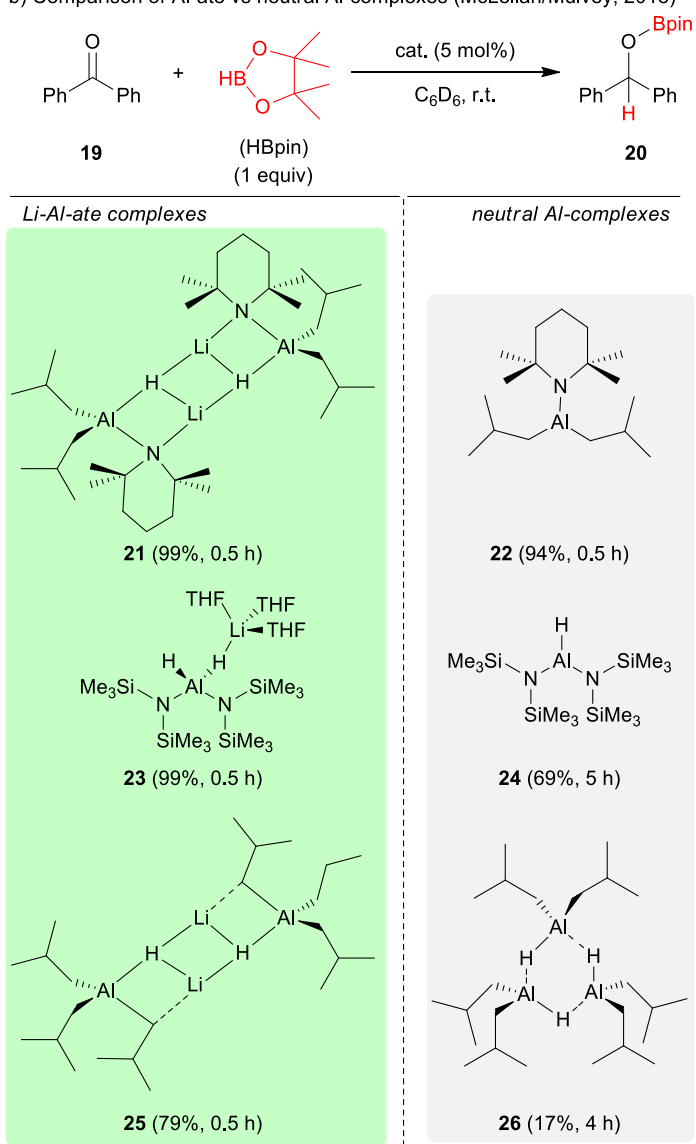

Scheme 5 Development of various Al-based hydroboration catalysts and comparison between neutral and anionic Al-complexes. 
Singh et al. reported a new stable and unique class of three coordinate and highly electrophilic cationic aluminium hydride (27) and aluminium methyl species (28) (Scheme 6a). ${ }^{51}$ The estimated Lewis acidity of these species is better compared to $\mathrm{B}\left(\mathrm{C}_{6} \mathrm{~F}_{5}\right)_{3}$ and $\mathrm{AlCl}_{3}$, making it an attractive choice for Lewis-acid mediated catalytic reactions. Catalytic efficacy was emphasised in the hydroboration reaction of aldehydes and ketones with high yields, demonstrating that the aluminium hydride cation is a better catalyst than its methyl counterpart. A proposed low coordinate intermediate (30) was successfully isolated and its characteristics determined for the first time. Its formation was identified as the overall rate-determining step of the hydroboration process (Scheme 6b).

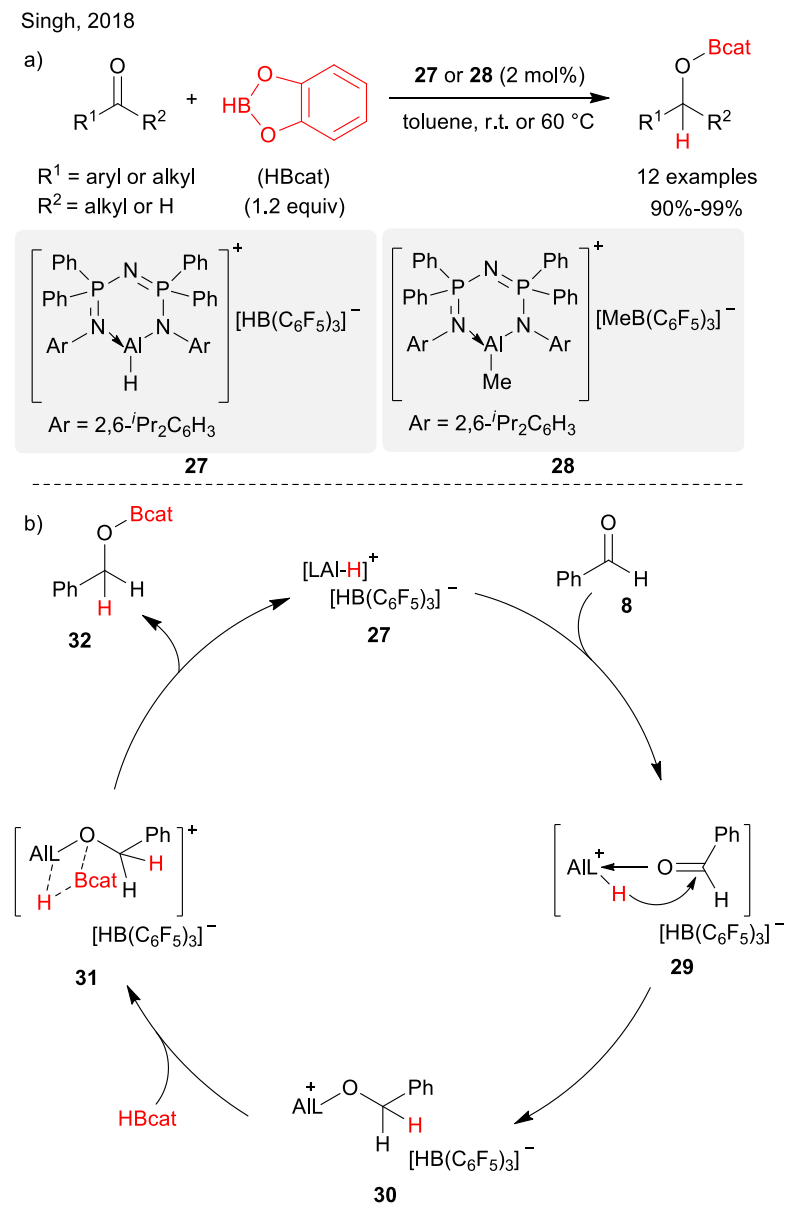

Scheme 6 Cationic Al-complex catalysed hydroboration of carbonyl compounds.

Yang et al. reported the synthesis of seven novel aluminium complexes supported by Schiff base ligands as catalysts in the hydroboration of carbonyl compounds (Scheme 7). ${ }^{52}$ These novel complexes were derived from $o$-diaminobenzene and $o$ aminothiophenol based Schiff base ligands and fully characterised using several spectroscopic techniques and X-ray crystallography. Compounds $\mathbf{3 3}$ and $\mathbf{3 4}$ were prepared from $o_{-}$ diaminobenzene containing backbone by the treatment with an equimolar amount of $\mathrm{AlMe}_{3}$ at $0{ }^{\circ} \mathrm{C}$. Compounds 35-39 were synthesised using a similar process, by reacting substituted $o$ aminothiophenol derivatives with $\mathrm{AlMe}_{3}$ or $\mathrm{H}_{3} \mathrm{Al} \cdot \mathrm{NMe}_{3}$ in an equimolar ratio. Compounds 35-39 delivered an interesting class of organic-inorganic hybrid structures bearing CNAISC elements that formed a five-membered ring. These complexes became precursors to the formation of new aluminium compounds. Al-complex $\mathbf{3 6}$ offered the highest activity in catalysing the hydroboration reaction compared to the rest. The first step of the mechanism is the formation of the Al-H bond when reacting with HBpin. A similar pathway was proposed as the next step of the mechanism (Schemes 5, 6), i.e., insertion of the $\mathrm{Al}-\mathrm{H}$ bond into aldehydes or ketones, resulting in the formation of the alkoxy Al-O bond. Subsequently, $\sigma$-bond metathesis with HBpin provided the desired hydroboration product along with regeneration of the active catalyst.
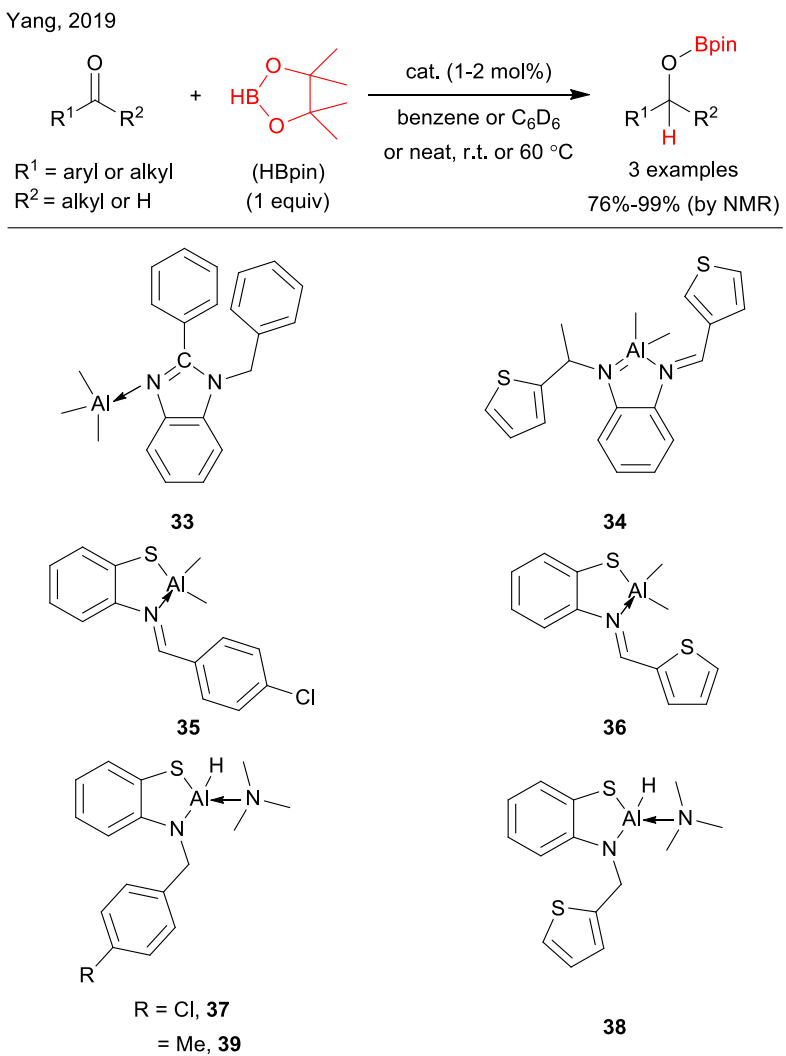

36

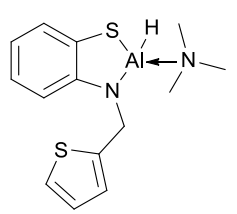

38

Scheme 7 Schiff base-supported Al-complex catalysed hydroboration of carbonyl compounds.

Zhang, Zheng and Dub et al. reported the selective dearomatisation of the Xtpy (terpyridine) ligand, resulting in the formation of zwitterionic Meisenheimer $\mathrm{Al}(\mathrm{III})$ complexes from hydroboration. ${ }^{53}$ Complex $\mathbf{4 0}$ was recognised as the most efficient pre-catalyst in generating high yields of products during the selective hydroboration of carbonyls as well as alkynes (Scheme 8a). Notably, the unique coordination mode of the Al centre was identified from the crystal structure of complex $\mathbf{4 0}$ (as shown in Scheme 8a). This reaction proceeded with a catalyst loading of a mere $0.1 \mathrm{~mol} \%$, which made it the most effective catalyst in hydroboration reactions reported to date. With such minimal levels of catalyst loading, turnover numbers (TONs) of the value of up to 1000 were obtained, which acknowledged complex $\mathbf{4 0}$ to be the most superior 
among various Al-complexes reported until then (Scheme 8b). Detailed theoretical calculations were performed to postulate the mechanism for catalytic hydroboration. Upon reaction with HBpin, pre-catalyst $\mathbf{4 0}$ furnished the 'possibly relevant' catalytic species $\mathrm{Al}$ (III) monohydride, or the 'more likely' dihydride complex $\mathbf{4 1}$ supported by ligands, demonstrating its 'chemically non-innocent' character (Scheme 8c).
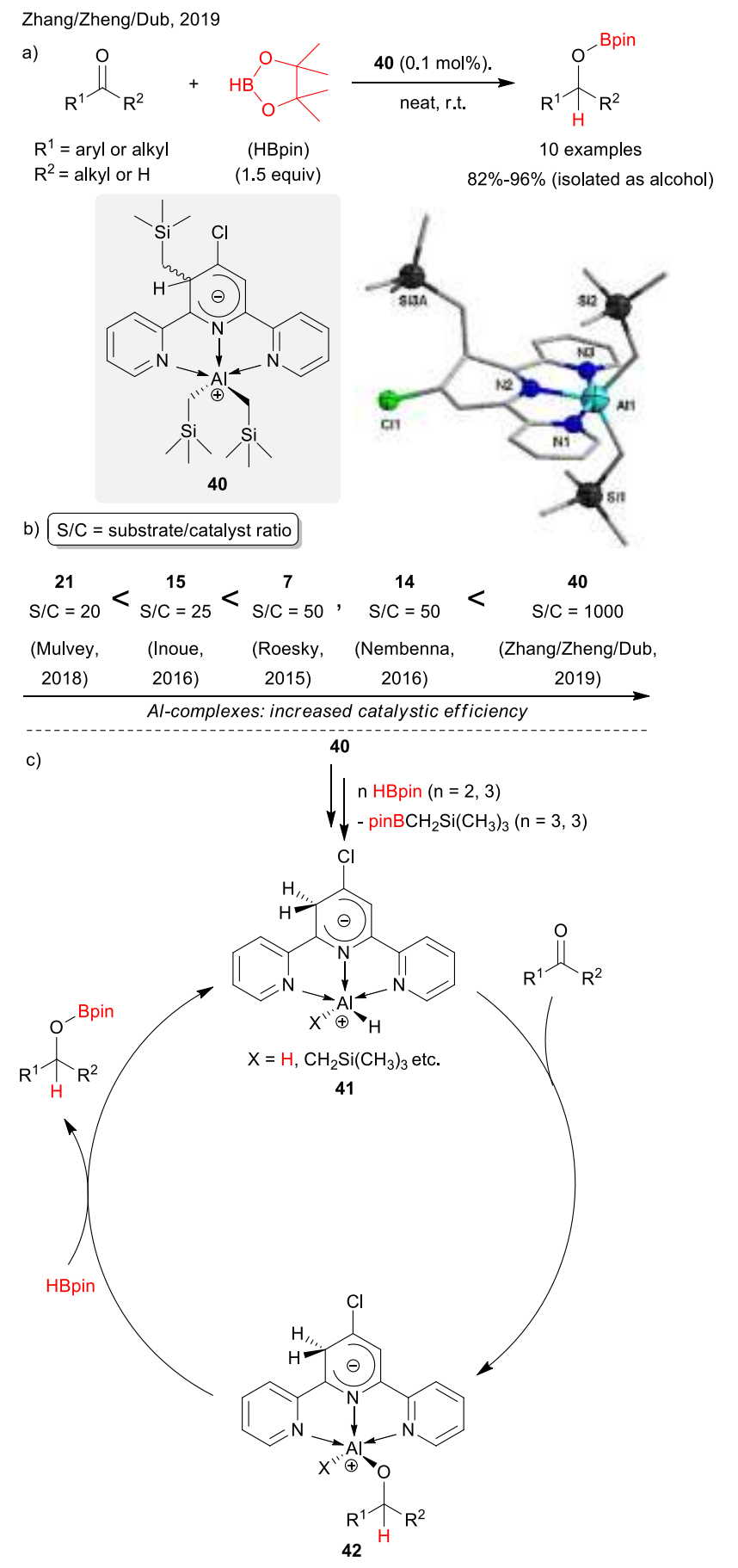

Scheme 8 Zwitterionic Meisenheimer Al-complex catalysed hydroboration of carbonyl compounds.

\subsection{Hydroboration of alkynes and alkenes}

Hydroboration of alkenes or alkynes demands of high interest as these borylated structural motifs are key building blocks for various organic syntheses. ${ }^{54-56}$ However, compared to the progress achieved in the hydroboration of aldehydes and ketones, it may be observed that fewer efforts have been made in similar transformations using $\mathrm{C} \equiv \mathrm{C}$ or $\mathrm{C}=\mathrm{C}$ bonds. It is noteworthy to mention that; alkenes and alkynes are of special interest as multiple products can be generated from the hydroboration reaction due to the lack of polarity of the unsaturated bonds. Hence, a suitable catalytic condition is required to obtain a certain product selectively (as shown in Scheme 9). Notably, aluminium complexes showed promising results in terms of selectivity issue for hydroboration of alkenes and alkynes by following anti-Markovnikov addition to produce predominantly the $\beta$-addition product.

a) Selective hydroboration of internal and terminal alkenes

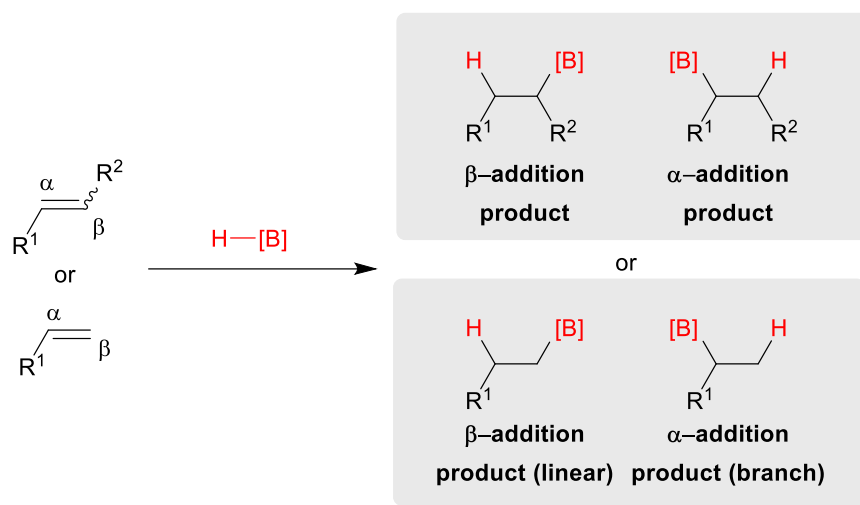

b) Selective hydroboration of internal and terminal alkynes

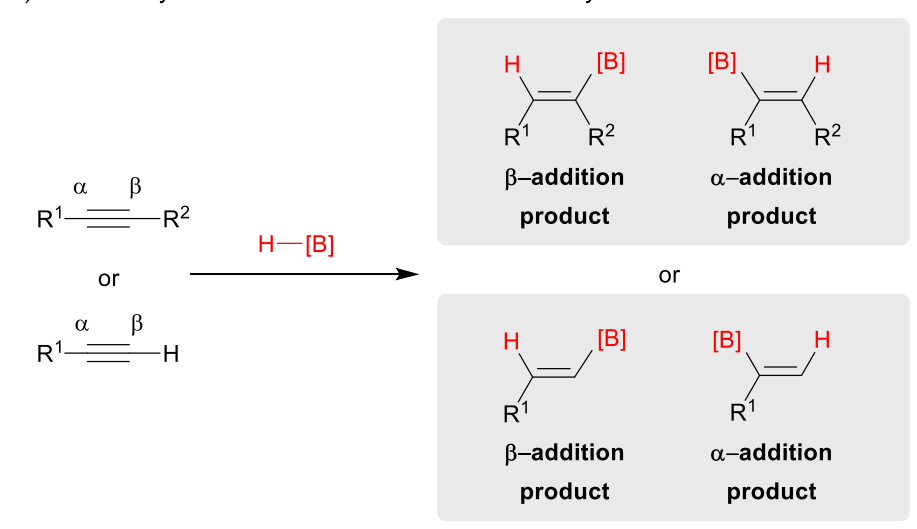

Scheme 9 Regioselective hydroboration of alkenes and alkynes under aluminium catalysed condition.

In this regard, another inventive work has come out as the first example of efficient and selective hydroboration of terminal alkynes using aluminium dihydride complex as catalyst $\mathbf{4 3}$ (Scheme 10) from Yang, Parameswaran, and Roesky et al. ${ }^{57}$ This group reported the reaction catalysed by aluminium dihydride complex $\mathbf{4 3}$ acting as a transition metal catalyst, as a subsequent result of their work on hydroboration of carbonyl compounds. ${ }^{31}$ The reaction afforded high yields with both aryland alkyl-substituted terminal alkynes, although it failed with 
internal alkynes (Scheme 10a). Deprotonation followed by a $\sigma$ bond metathesis pathway was proposed as the plausible reaction mechanism (Scheme 10b). In the first step, catalyst $\mathbf{4 3}$ undergoes deprotonation with alkynes to generate compound 45 with the evolution of $\mathrm{H}_{2}$ gas. Insertion of HBpin into intermediate $\mathbf{4 5}$ occurs in a syn-fashion. In the final step, $\sigma$-bond metathesis takes place with alkenyl aluminium species $\mathbf{4 6}$, yielding a protonated hydroboration product 47 with the regeneration of the active catalytic component $\mathbf{4 5}$. A detailed theoretical calculation revealed the generation of aluminium acetylide (45) to be the overall rate-determining step of the process.
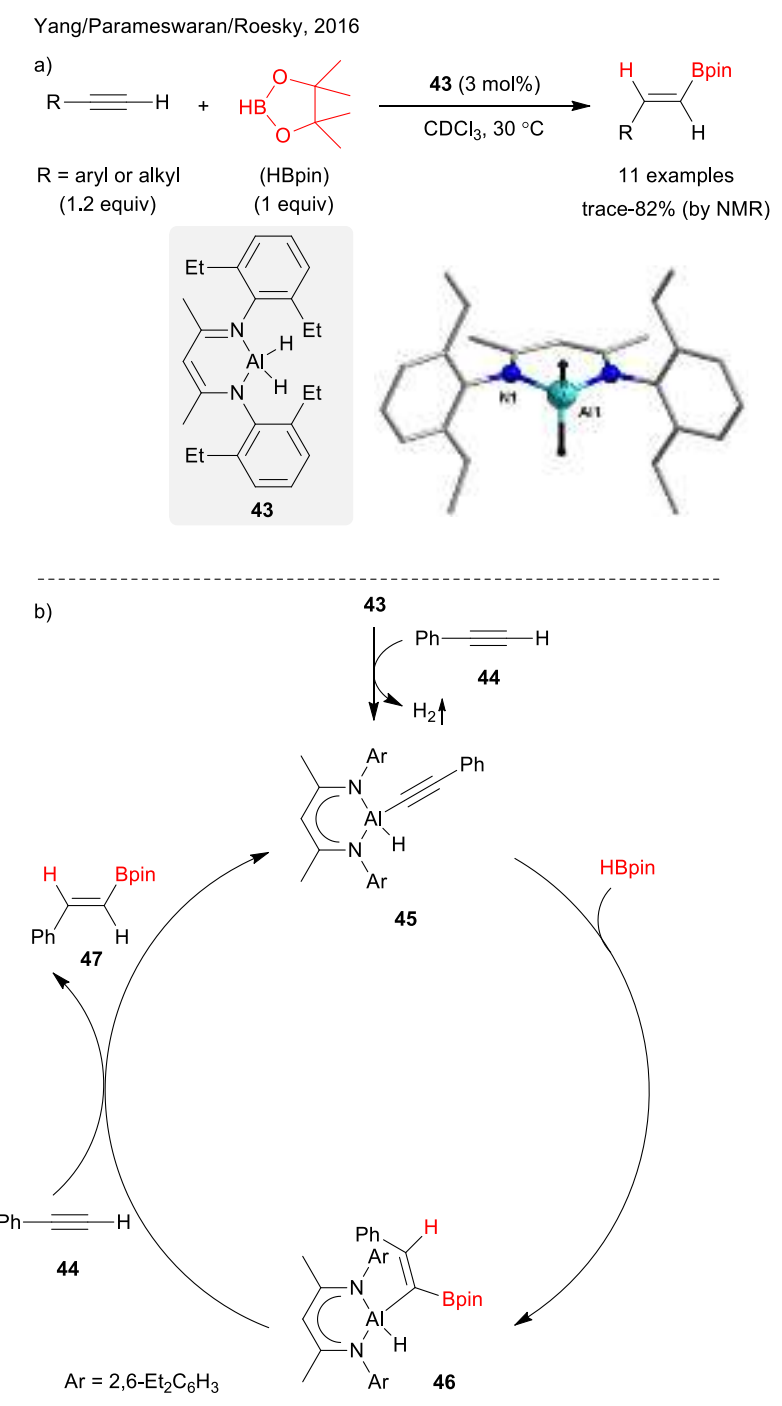

Scheme $10 \beta$-diketimine ligated Al-complex catalysed hydroboration of alkynes.

Inspired by the Roesky group's discovery, Inoue et al. reported that dimeric aluminium hydrides (48 or 49), supported by $\mathrm{NHI}$ ( $N$-heterocyclic imine), could catalyse the selective hydroboration of terminal alkynes (Scheme 11). ${ }^{46}$ Only three examples of alkynes were presented to demonstrate the catalytic efficiency of the aluminium complexes. A distinct electronic effect was realised with these three electronically different substituted alkynes, where electronically neutral phenylacetylene (47) reacted faster than the electron-deficient alkyne (50). Further, an electron-rich substrate (51) showed a significantly faster reaction rate compared to substrate $\mathbf{5 0}$. The same reaction mechanism was proposed as the one explained by Roesky and co-workers (Scheme 10b).
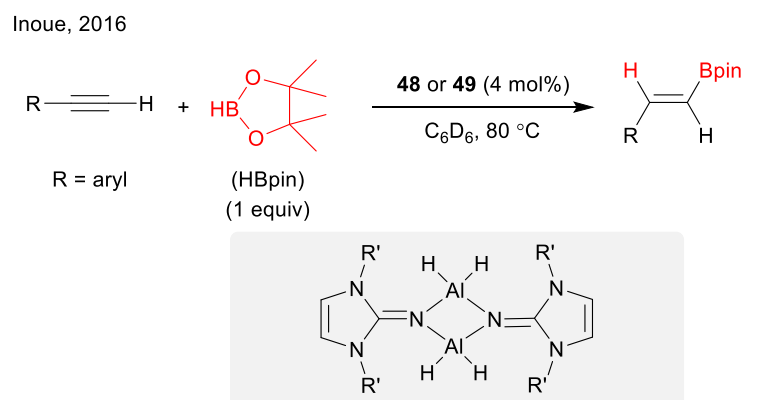

$\mathrm{R}^{\prime}=$ 2,4,6-trimethylphenyl (Mes), 48 (4 mol\%)

= 2,6-diisopropylphenyl (Dipp), 49 (4 mol\%)

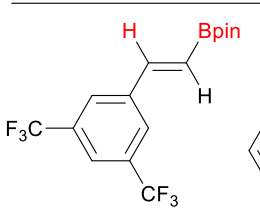

$5040 \%$

(using $48,40 \mathrm{~h}$ )

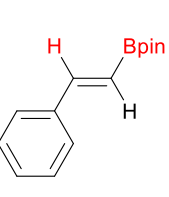

$4780 \%$

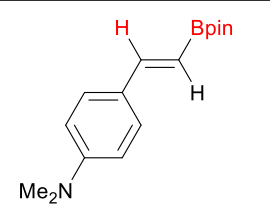

$\mathbf{5 1 5 0 \%} \quad \mathbf{5 1} 19 \%$ (using 48, $40 \mathrm{~h}$ ) (using 48, $12 \mathrm{~h}$ ) (using $49,12 \mathrm{~h}$ )
Scheme $11 \mathrm{NHI}$-supported dimeric Al-complex catalysed hydroboration of alkynes.

Thomas and Cowley et al. reported the unprecedented catalytic use of commercially available Al-hydride (DIBAL-H) or stable alkyl aluminium ( $\left.\mathrm{Et}_{3} \mathrm{Al} \cdot \mathrm{DABCO}\right)$ in the hydroboration reaction between alkynes and HBpin (Scheme 12).58 This work was promising since they were able to catalyse internal alkynes which were not obtained during previous studies using aluminium catalysts (Scheme 12a). For unsymmetrically substituted internal alkynes, the $\beta$-addition product was formed predominantly over the $\alpha$-addition product (see, Scheme $9 \mathrm{~b}$ and Scheme 12a). The authors explained a working mechanism that was different from the one suggested by the Roesky ${ }^{57}$ and Inoue groups. ${ }^{46}$ Based on the outcomes of their experiment, they refuted the involvement of a deprotonation step in the reaction between terminal alkynes and the catalyst as the first step of the mechanism (as mentioned in Scheme 10b), which was supported by the successful reaction with internal alkynes (Scheme 12b). Moreover, the authors did not observe the evolution of any hydrogen gas from the reaction with a terminal alkyne. On the other hand, the generation of aluminium hydride (53) as the active catalyst from alkyl aluminium (52) was believed to proceed from the reaction with HBpin, which was also used as the hydride source. Next, the insertion of an alkyne into aluminium hydride (53) in a syn-orientation promoted the formation of a hydroalumination product (54). $\sigma$-bond metathesis with HBpin (Al to B exchange) in the final step was also recognised as the rate-limiting step, resulting in the hydroboration product along with regeneration of the aluminium hydride catalyst (53). 
Simple aluminium hydride pre-catalysts, such as commercially available $\mathrm{LiAlH}_{4}$ or sodium bis(2-methoxyethoxy)aluminium hydride (red-Al) efficiently catalyse the selective hydroboration of alkenes (Scheme 13a) to provide the $\beta$-addition product up to $99 \%$ selectivity (see, Scheme 9 a and Scheme 13a). ${ }^{59}$ After optimisation, the use of pyrophoric $\mathrm{LiAlH}_{4}$ was lowered to operationally safe limits (10 mol\%) by Cowley, Thomas and coworkers. With this, one of the most easily accessible sources of aluminium catalysts could be used in hydroboration reactions using HBpin as a source of boron. Interestingly, hydroboration of other unsaturated compounds was achieved with significantly low catalyst loading, such as $0.5 \mathrm{~mol} \%$ for ketone (55) and ester (56) and 1 mol\% for nitrile (57) (Scheme 13b). A suitable example also demonstrated the selective functionalisation of ketones over esters using the catalyst $\mathrm{LiAlH}_{4}$ (Scheme 13c).

\section{Thomas/Cowley, 2016}

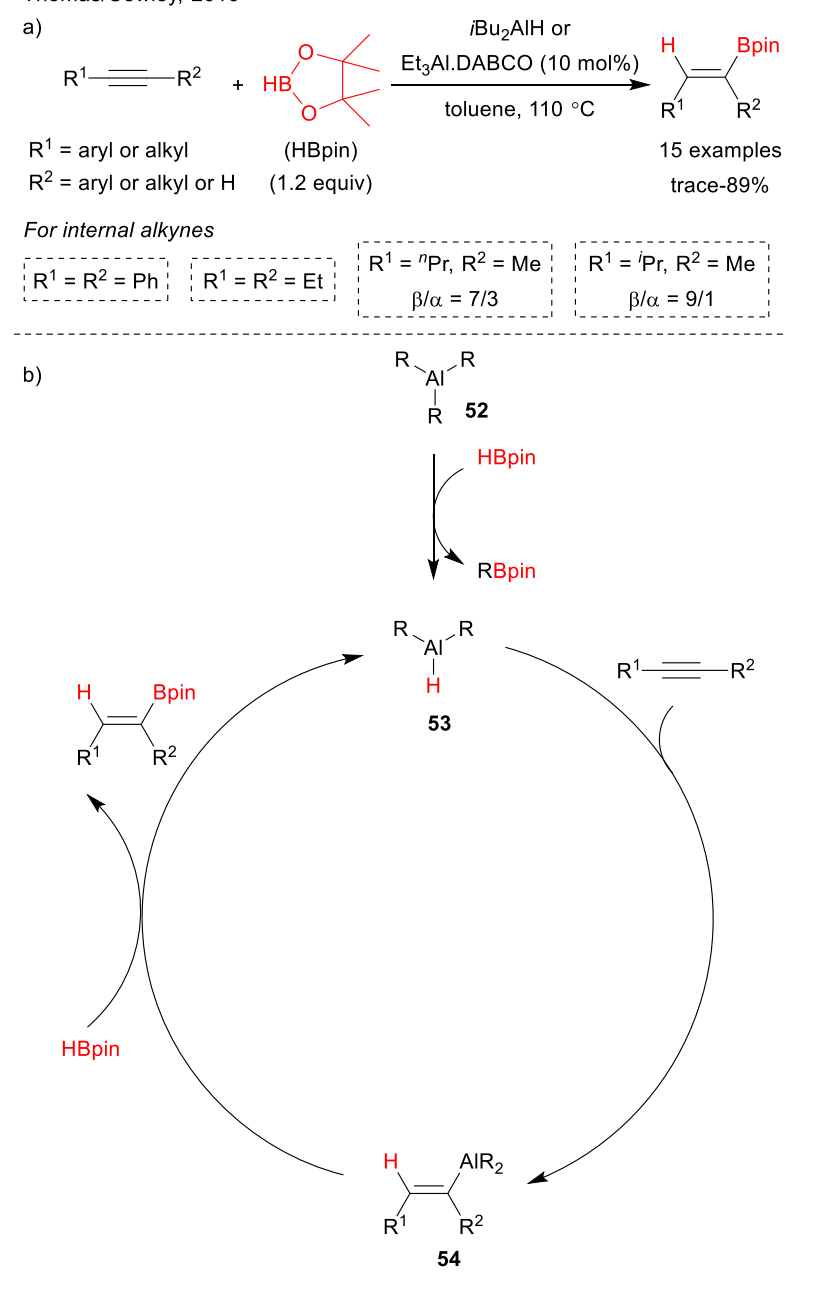

Scheme 12 DIBAL-H or Et ${ }_{3}$ Al-DABCO catalysed hydroboration of alkynes.

Cowley/Thomas, 2018

a)

$$
\mathrm{R}^{1}=\text { aryl or alkyl }
$$

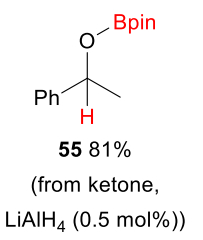

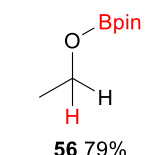

$679 \%$

(from ester, $\left.\mathrm{LiAlH}_{4}(0.5 \mathrm{~mol} \%)\right)$

$$
{ }_{\left.\mathrm{LiAlH}_{4}(1 \mathrm{~mol} \%)\right)}^{\mathbf{5 7 7 1 \%}}
$$

$\mathrm{Ar}=4$-trifluoromethylbenzene

c)

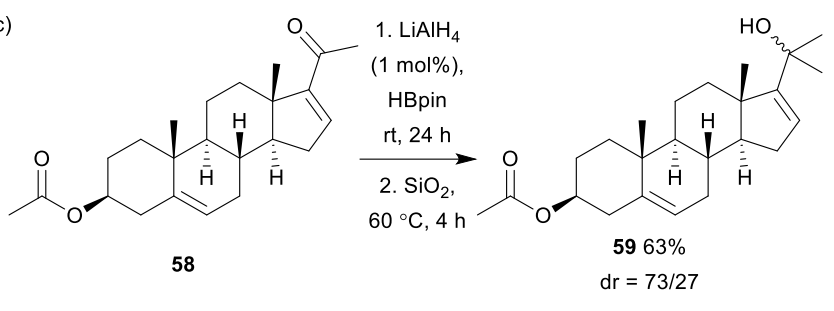

Scheme $13 \mathrm{LiAlH}_{4}$ catalysed hydroboration of alkenes and expanding scope with carbonyls and nitrile with low catalyst loading.

McLellan and Mulvey et al. reported their synthesis of a bimetallic Li-Al-ate complex as well as a neutral Al-complex in the catalytic hydroboration of alkynes (Scheme 14). ${ }^{49}$ The authors presented a comparative study between several aluminium catalysts in the reactivity of terminal and internal alkynes (Scheme 14a), similar to the one performed using carbonyl compounds. According to the outcomes of their experiment, bimetallic complexes (21 and 25) with Li-Al cooperative interaction demonstrated superior reactivity compared to the only aluminium-bearing complex (22) and this finding was well suited with phenyl acetylene (47). However, with internal alkyne 60, complexes $\mathbf{2 1}$ and $\mathbf{2 5}$ failed to provide efficient reactivity compared to complex $\mathbf{2 6}$. This inconsistency in reactivity amongst aluminium-based complexes can be explained through the steric effect (Scheme 14b). On the other hand, internal alkynes bearing smaller methyl (-Me) groups again succeeded in supporting the original catalytic superiority of the lithium aluminates. In the case of an unsymmetrically substituted internal alkyne $\beta$-addition product was obtained as major product (see, Scheme $9 \mathrm{~b}$ and Scheme 14a). Okuda and Mulvey et al. also reported the aluminium catalysed hydroboration with only terminal alkynes. ${ }^{47}$ 
McLellan/Mulvey, 2018

a)

$$
\text { (t) cat. (10 mol\%) }
$$
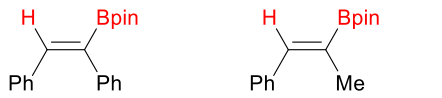

cat. $21(71 \%), 22(0 \%)$, cat. $21(0 \%), 26(40 \%)$ cat. $25(60 \%, \beta / \alpha=2.2 / 1)$, $25(83 \%), 26(85 \%) \quad 25(10 \%), \quad 26$ (trace)

b) Acetylene insertion: explanation via steric effect

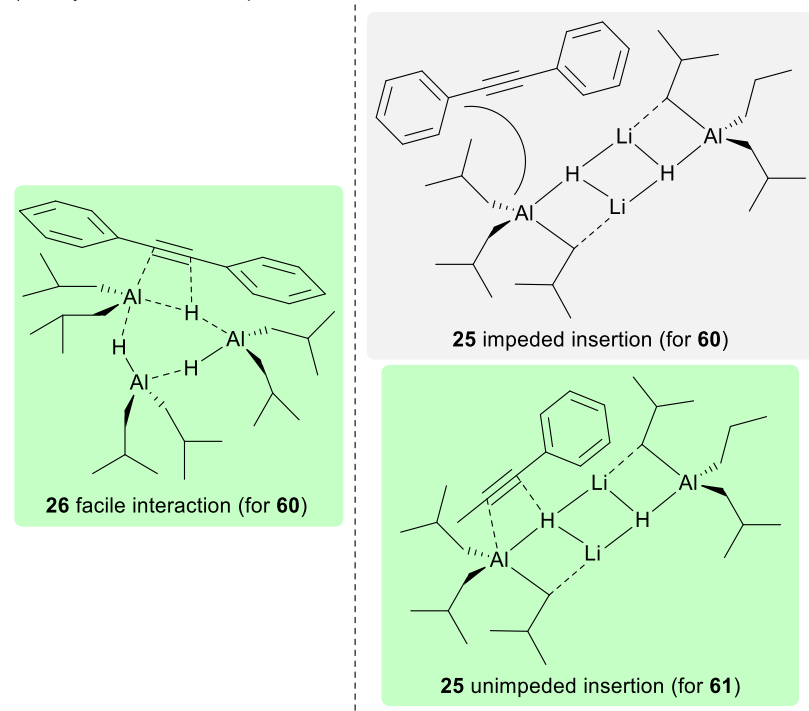

Scheme 14 Neutral Al-complex and Li-Al-ate complex catalysed hydroboration of alkynes.

Hydroboration of terminal and internal alkynes catalysed by the zwitterionic Meisenheimer Al-complex (40) catalysed was reported by Zhang, Zheng and Dub et al. (Scheme 15a). ${ }^{53}$ The reaction proceeded with a high TON value of up to 1000 using phenylacetylene in a highly syn-selective manner. Based on the substrate to catalyst $(\mathrm{S} / \mathrm{C})$ value, this aluminium complex showed considerable efficiency compared to previously known catalysts in the hydroboration of alkynes. The proposed mechanism (Scheme 15b) supported the insertion of alkynes into the Al-catalyst $\mathbf{4 1}$ to form the syn-hydroalumination intermediate $\mathbf{6 2}$ followed by $\sigma$-bond metathesis with HBpin to generate the product.
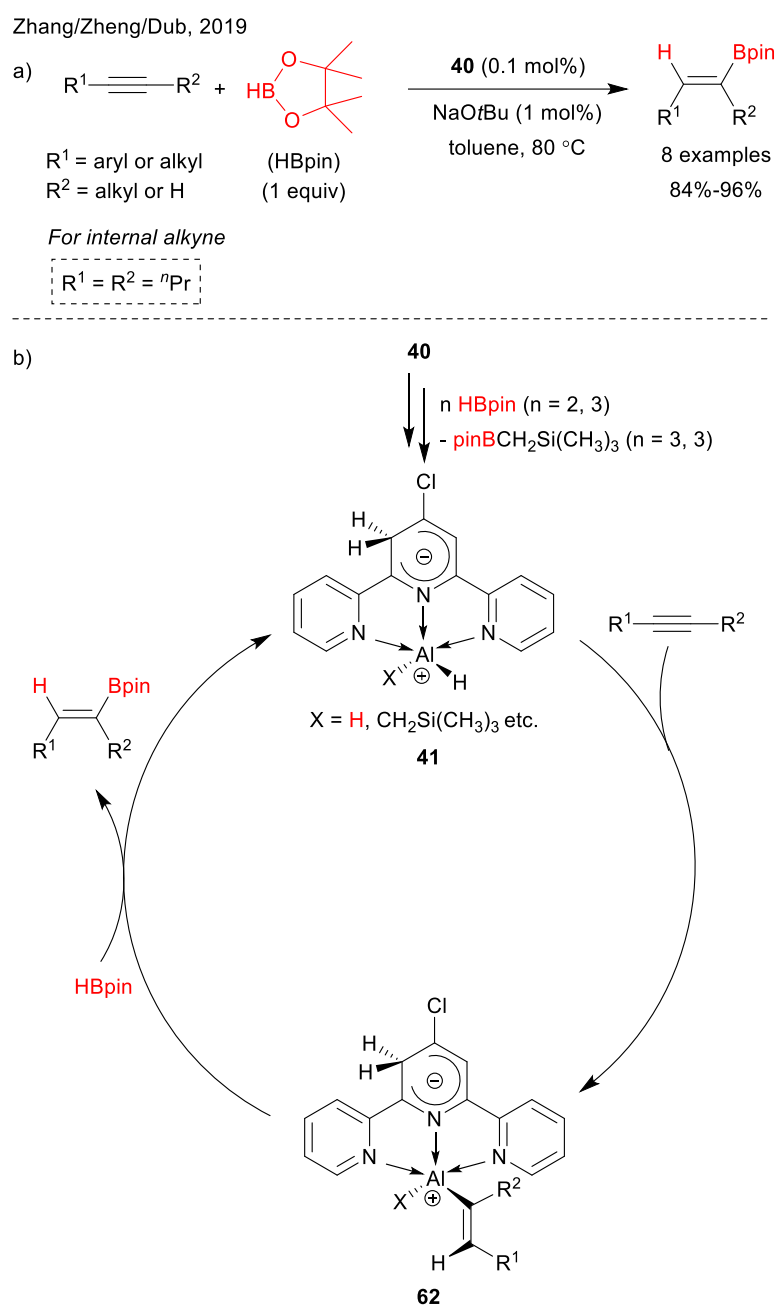

Scheme 15 Zwitterionic Meisenheimer Al-complex catalysed hydroboration of alkynes.

The working group of Hong, $\mathrm{Xu}$ and Shi developed a mechanism for the efficient and selective hydroboration of alkenes and alkynylsilanes using an alkyl aluminium catalyst (Scheme 16a). ${ }^{60}$ The corresponding boronates were obtained with high regioselectivity and an established mechanism involving

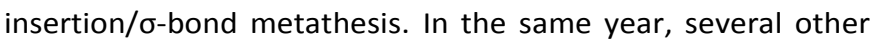
research groups reported the hydroboration of alkenes and alkynes using aluminium complexes. Panda et al. demonstrated another method to obtain alkyl or alkenyl boronic esters from terminal alkenes or alkynes at room temperature using a functionalised amidophosphine ligated Al-complex 63 (Scheme 16b). ${ }^{61}$ This protocol benefited from the formation of a large array of selective hydroboration products. Another commercial source of the aluminium hydride catalyst, i.e., lithium diisobutyl-tert-butoxyaluminium hydride (LDBBA) (64) revealed catalytic activity in hydroboration of terminal alkenes by An et al. (Scheme 16c). ${ }^{62,63}$ Further, the application of borylated product in the formation of various synthetically important synthons was presented. Very recently, Nembenna and coworkers synthesised a conjugated bis-guanidine (CBG)supported aluminium dihydride $\mathbf{6 5}$ for use in the selective hydroboration of alkynes (Scheme 16d). ${ }^{64}$ In the case of an 
unsymmetrically substituted internal alkyne, selective formation of the $\beta$-addition product was reported (see, Scheme $9 \mathrm{~b}$ and Scheme 16d). Furthermore, for the first time, chemoselective functionalisation of alkynes and nitriles over other unsaturated compounds was well depicted by the authors.

a) Hong/Xu/Shi, 2019

$$
\begin{array}{ll}
\text { or } \\
\mathrm{R}^{1}=\text { aryl or alkyl } \\
\mathrm{R}^{2}=\text { alkyl, } \mathrm{R}^{3}=\text { aryl or alkyl }
\end{array}
$$
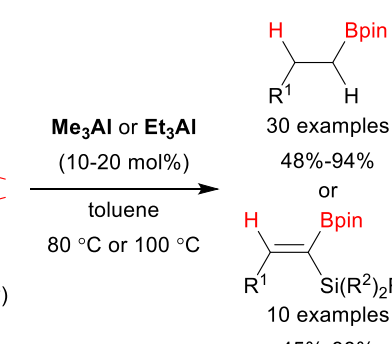

30 examples

$48 \%-94 \%$

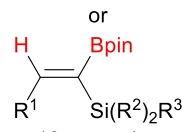

10 examples

$45 \%-88 \%$

b) Panda, 2019
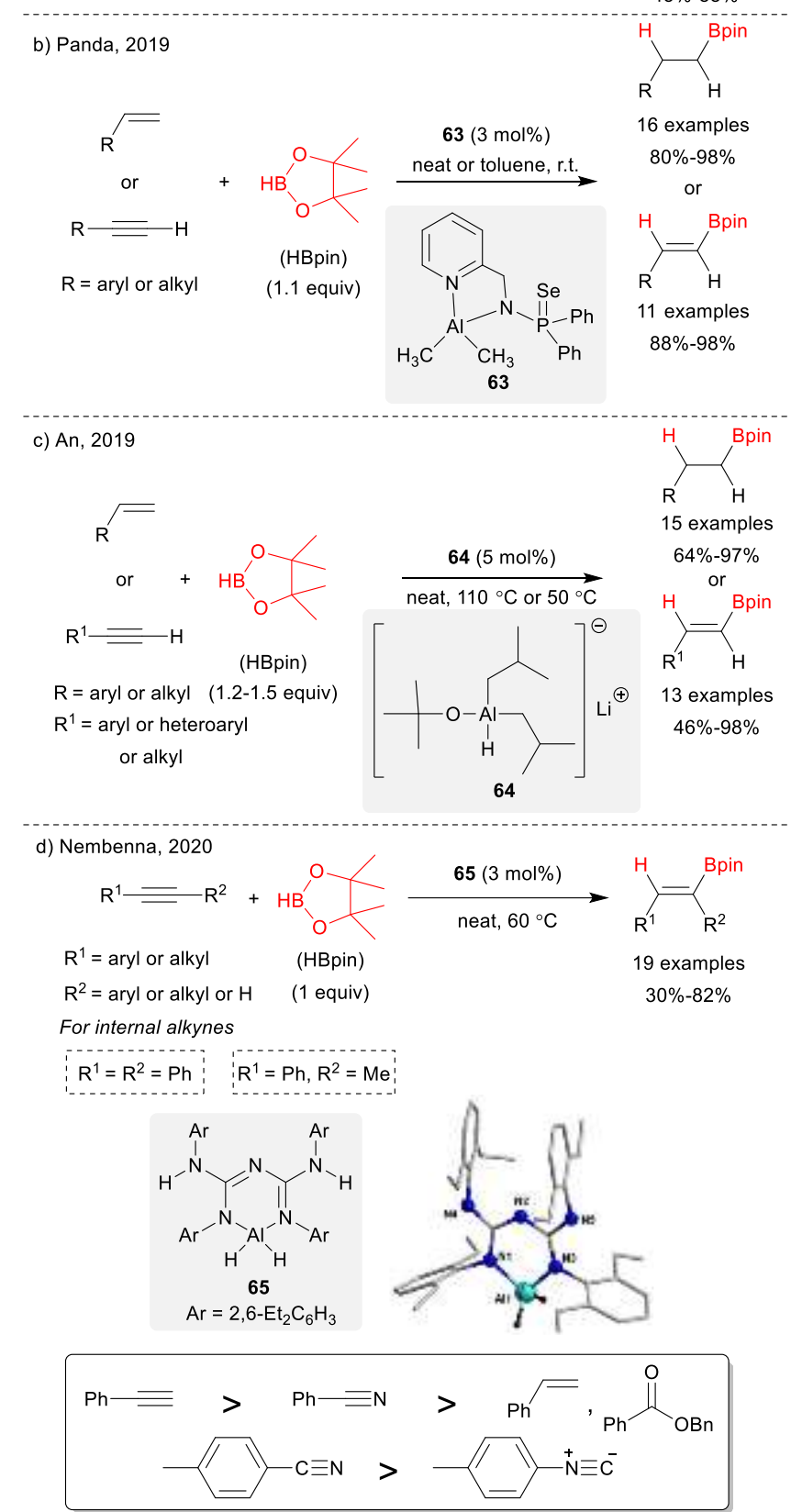

Scheme 16 a) Alkyl aluminium, b) amidophosphine ligated Al-complex, c) LDBBA and d) CBG-supported Al-complex catalysed hydroboration of alkenes or alkynes.
Very recently, Carmalt and Bakewell et al. reported the hydroboration of terminal alkynes catalysed by a class of aluminium amidinate hydride and alkyl complexes. ${ }^{65}$ Elucidation of reaction mechanism proposed the formation of an Al-borane adduct in the initial step which is different from the previously proposed ones involving aluminium acetylide or hydroalumination as the first step.

\subsection{Hydroboration of imines}

Selective hydroboration of imines was reported by McLellan and Mulvey et al. in the year 2018 using anionic aluminium-ate complexes (21, 23, 25) (Scheme 17). ${ }^{49}$ In general, heterobimetallic lithium aluminates were more superior catalytic systems than the corresponding neutral aluminium species. An important comparison on this was also presented (Scheme 17a). The reaction mechanism involves deprotonation of imine 68 to provide the active catalytic species 69 by coordination with the Al-centre (Scheme 17b). In the next step, hydroboration proceeds to provide $\mathbf{7 0}$ following protonation to regenerate the active catalyst $\mathbf{6 9}$ along with delivering the hydroboration product $\mathbf{6 7}$. An et al. described a suitably mild condition for the regioselective hydroboration of imines using lithium diisobutyl-tert-butoxyaluminium hydride (LDBBA, 64), which resulted in the formation of secondary amines (Scheme 18a). ${ }^{63}$ Lithium coordination to the anionic aluminate was found to play a prominent role in accelerating the hydride transfer during the hydroboration process. Conjugated bis-guanidine (CBG) supported aluminium dihydride complex 65 reported by Nembenna group was effective in delivering a few examples of the hydroboration of imines (Scheme 18b). ${ }^{64}$ 


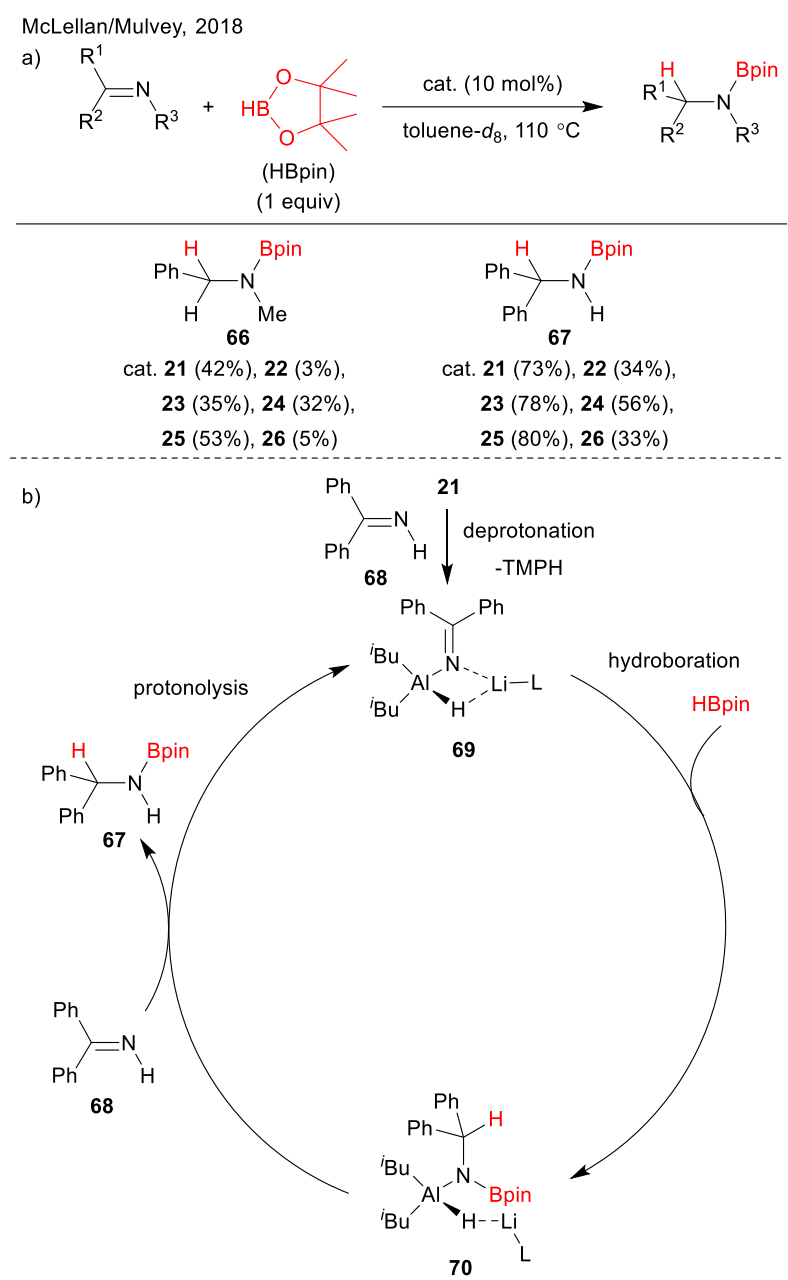

Scheme 17 Neutral Al-complex and Li-Al-ate complex catalysed hydroboration of imines.

a) An, 2019

\begin{tabular}{|c|c|c|c|}
\hline $\mathrm{R}^{2}$ & & 64 (5 mol\%) & $R^{2}$ \\
\hline & & neat, $50^{\circ} \mathrm{C}$ & $\mathrm{R}^{1}$ \\
\hline $\mathrm{R}^{1}=$ aryl & (HBpin) & & 9 examples \\
\hline $\mathrm{R}^{2}=$ aryl & (1.5 equiv) & & $78 \%-99 \%$ \\
\hline
\end{tabular}

b) Nembenna, 2020

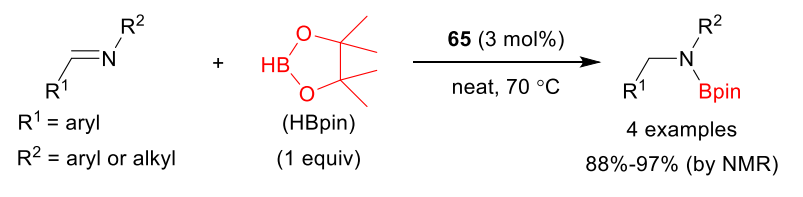

Scheme 18 a) LDBBA and b) CBG-supported Al-complex catalysed hydroboration of imines.

\subsection{Hydroboration of nitriles}

Panda et al. reported the first example of aluminium catalysed chemo-selective hydroboration of several aryl or alkyl nitriles (Scheme 19a). ${ }^{66}$ Aluminium alkyl complex $\mathbf{7 1}$ is used as a precatalyst and generates the active aluminium hydride $\mathbf{7 2}$ by reacting with HBpin in the first step of the mechanism (Scheme 19b). In the following step, $\sigma$-bond metathesis between the nitrile and aluminium hydride takes place, forming the aluminium coordinated imine intermediate 75 . In the subsequent steps, two molecules of HBpin react, one by one, to finally furnish the double hydroboration product 79 and the active catalyst is regenerated. The step from $\mathbf{7 5}$ to $\mathbf{7 6}$ was considered the rate-limiting step. Ma, Yang and co-workers reported that aluminium catalyst $\mathbf{4 3}$ catalysed the hydroboration of nitriles under mild and solvent-free conditions (Scheme 20a). ${ }^{67}$ Designing the ligand appropriately by addressing both steric and electronic concerns, $\beta$-diketiminato ligated aluminium complex $\mathbf{8 0}$ efficiently triggers the reaction. Synthesis of the alkyl aluminium complex $\mathbf{8 0}$ from compound $\mathbf{4 3}$ and its utility in catalysing the double hydroboration of nitriles was demonstrated by the Ma, Yang and Roesky group (Scheme 20b). ${ }^{68}$ A mechanism similar to the one by Panda et al. was proposed. Nembenna et al.'s aluminium dihydride complex 65 offers efficient hydroboration of various aryl and alkyl nitriles (Scheme 20c). ${ }^{64}$ Moreover, this catalyst with low catalyst loading and mild reaction conditions was capable of performing the hydroboration of several other functionalities such as imines, alkenes, alkynes, pyridine, carbodiimide and isocyanide. 
Panda, 2019

a)

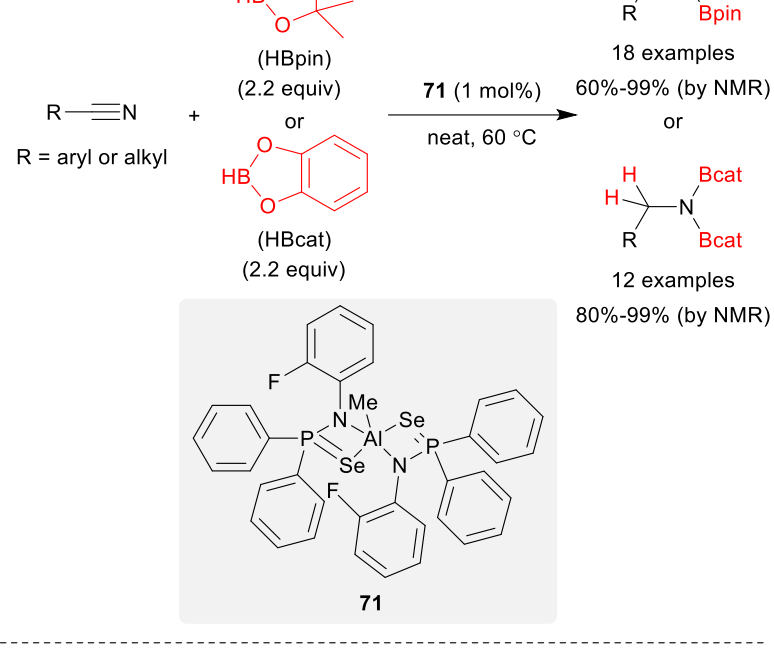

b)

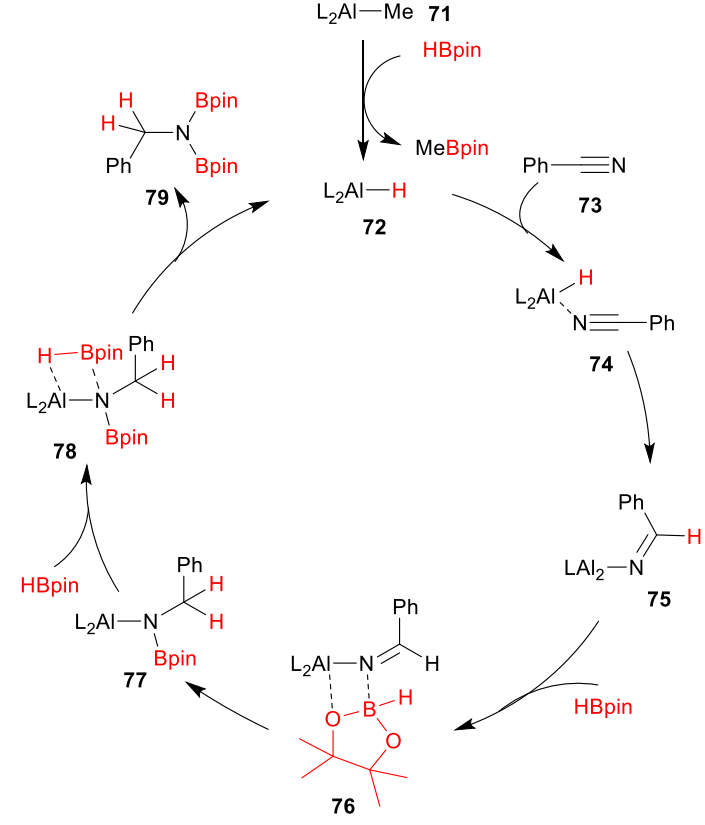

Scheme 19 Alkyl Al-complex catalysed hydroboration of nitriles. a) Ma/Yang, 2019

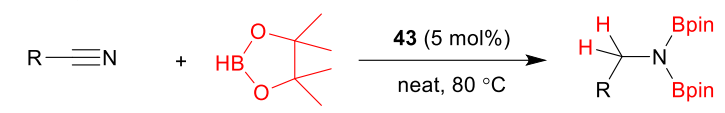

$\mathrm{R}=$ aryl or alkyl

$$
\begin{gathered}
\text { (HBpin) } \\
\text { (2.1 equiv) }
\end{gathered}
$$

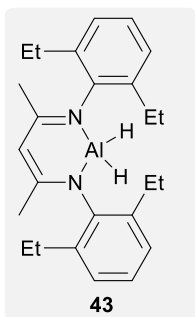

11 examples $61 \%-99 \%$ (by NMR)

b) Ma/Yang/Roesky, 2019

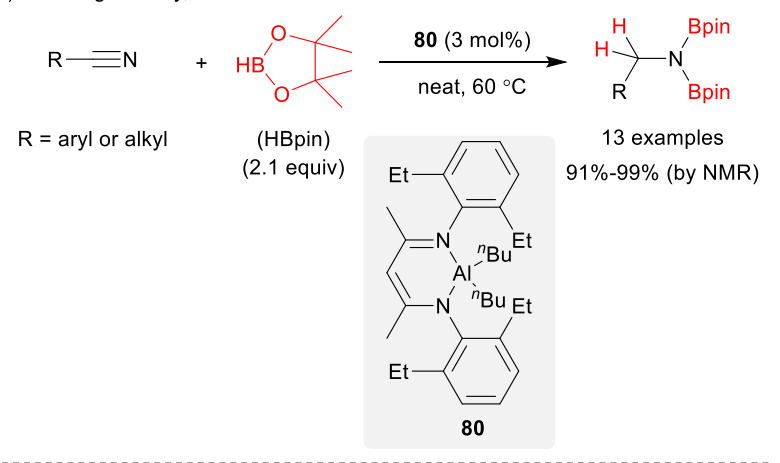

c) Nembenna, 2020

$$
\mathrm{R}=\text { aryl or alkyl } \underset{\substack{\text { (HBpin) } \\ \text { (2 equiv) }}}{\mathrm{R}}=\mathrm{N5(3 \textrm {mol } \% )}
$$

Scheme 20 a) and b) $\beta$-diketimine ligated Al-complex, c) CBG-supported Alcomplex catalysed hydroboration of nitriles.

\subsection{Hydroboration of carbodiimides}

Selective mono hydroboration of carbodiimides was achieved for the first time by Ma, Yang and Roesky et al. (Scheme 21a). ${ }^{69}$ Several $N, N$-bidentate ligand coordinated aluminium hydride complexes were utilised as proficient catalysts in this transformation. Interestingly, with prolonged heating, the $\mathrm{N}$ borylated product $\mathbf{8 2}$ was converted to the C-borylated compound 83 (Scheme 21 b). While catalysts $\mathbf{4 3}$ and 84 provided a mixture of both $\mathrm{N}$ - and C-borylated products, catalyst 85 facilitated the generation of C-borylated products in $100 \%$ selectivity. A possible explanation for the formation of compound $\mathbf{8 3}$ over time is the higher electron density of the $\mathrm{C}=\mathrm{N}$ bond, compared to the $\mathrm{C}-\mathrm{N}$ bond, which eases the shifting of the electrophilic Bpin moiety towards the double bond, thus forming a $\mathrm{C}-\mathrm{B}$ bond. The mechanism follows with the insertion of aluminium hydride $\mathbf{4 3}$ into the $\mathrm{C}=\mathrm{N}$ bond in a syn-orientation fashion to form $\mathbf{8 8}$ (Scheme 21c). Next, HBpin participates in the reaction via $\sigma$-bond metathesis, and exchange of aluminium by Bpin proceeds at the $N$-centre to give the product 82 and regenerate the active catalyst $\mathbf{4 3}$. Alkyl aluminium complex $\mathbf{8 0}$ reported by the Ma, Yang and Roesky group also catalysed the selective single hydroboration of carbodiimides (Scheme 22a). ${ }^{68}$ Examples of selective single site hydroboration of carbodiimides 
were presented by Nembenna et al. using aluminium catalyst 65 with quantitative conversion (Scheme 22b). ${ }^{64}$

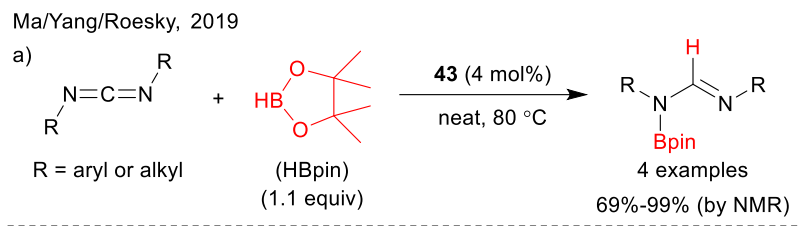

b) Formation of C-borylformamidine product

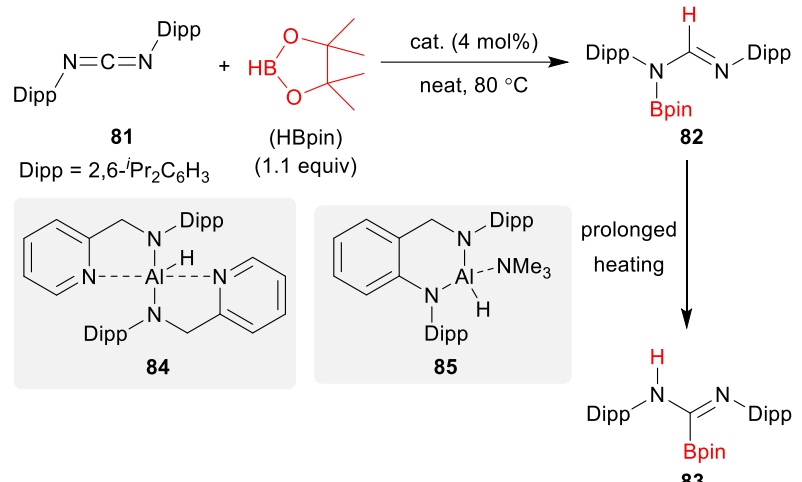

cat. 43: $100 \%$ conversion $(\mathbf{8 2} / \mathbf{8 3}=2 / 8)$

cat. $84: 44 \%$ conversion $(82 / 83=3 / 1)$

cat. 85 : $60 \%$ conversion (only 83 )

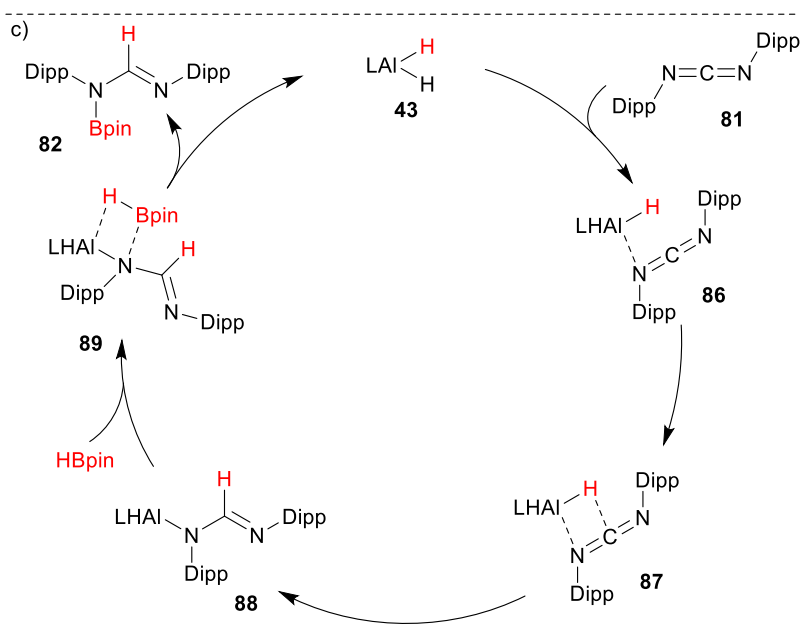

Scheme $21 \mathrm{~N}, \mathrm{~N}$-bidentate ligated Al-complex catalysed hydroboration of carbodiimides.

a) Ma/Yang/Roesky, 2019

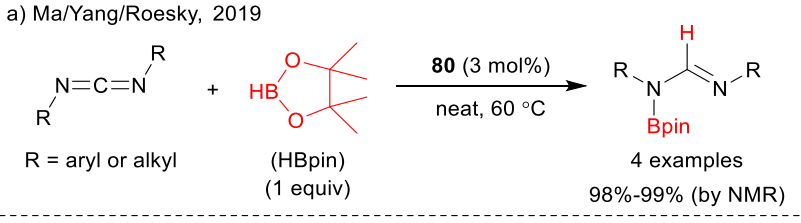

b) Nembenna, 2020

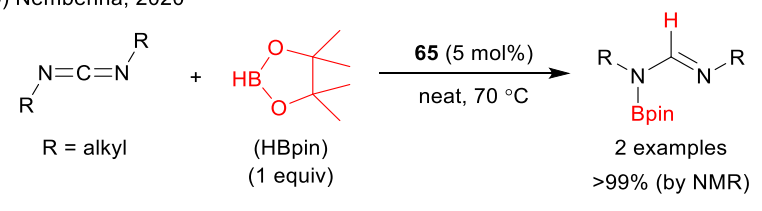

Scheme 22 a) $\beta$-diketimine ligated Al-complex and b) CBG-supported Al-complex catalysed hydroboration of carbodiimides.

\subsection{Hydroboration of carbon dioxide}

Mézailles and So et al. reported the use of a bis(phosphoranyl)methanido aluminium hydride for the catalytic hydroboration of $\mathrm{CO}_{2}$ for the first time (Scheme 23)..$^{70}$ By using catalyst $\mathbf{9 0}$ and $\mathrm{BH}_{3} \cdot \mathrm{SMe}_{2}$ as hydroboration reagent, extremely pure catalytic reaction was taken place towards the synthesis of trimethyl borate (91) within an hour in excellent yield. Mechanistic studies revealed the formation of $\mathrm{Al}-\mathrm{O}$ bond in complex $\mathbf{9 0}$ by the activation of $\mathrm{CO}_{2}$ which then resulted into the formation of 91 and 92 by subsequent reaction with $\mathrm{BH}_{3} \cdot \mathrm{SMe}_{2}$ and regeneration of catalyst 90 . This catalyst also showed activity for the hydroboration of carbonyls, alkynes and nitriles.

$$
\begin{aligned}
& \text { Mézailles/So, } 2021
\end{aligned}
$$

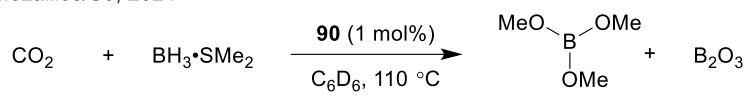

$$
\begin{aligned}
& \begin{array}{lllll}
\text { (1 bar) (1 equiv) } & \mathrm{Ph} & \mathrm{Cl} & 91 & 92
\end{array} \\
& \mathrm{Mes}^{-} \\
& 90
\end{aligned}
$$

Scheme 23 bis(phosphoranyl)methanido aluminium hydride catalysed hydroboration of carbon dioxide.

\subsection{Hydroboration of pyridine}

The only example of selective hydroboration of heterocycle (pyridine) was achieved using the aluminium catalyst 65 synthesised by Nembenna and co-workers (Scheme 24). ${ }^{64}$ The reaction proceeded under solvent-free and sufficiently mild conditions to achieve the hydroboration reaction in nearly full conversion.

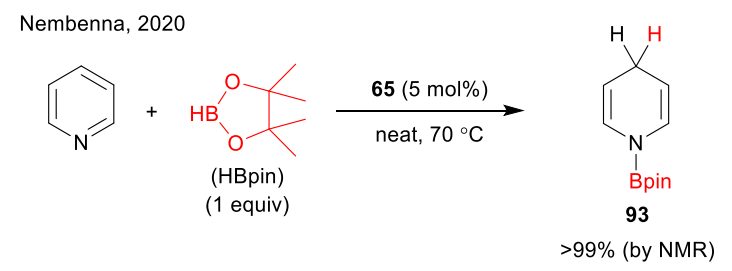

Scheme 24 CBG-supported Al-complex catalysed hydroboration of pyridine.

\section{Conclusions}

This Frontiers review summarises developments in the field of aluminium catalysed hydroboration of reducible functionalities. To briefly recapitulate, the importance of aluminium as a catalyst is its high abundance in the earth's crust, comparable efficiency as a transition metal catalyst (with relatively less expense) and greater appeal from the perspective of sustainability. The pioneering work of the Roesky group was the first example of an aluminium complex catalysed hydroboration reaction, as reported in 2015 . They proposed a 1,3-diketimine ligated Al-complex to efficiently catalyse the hydroboration of several aldehydes and ketones. Subsequently, various 
aluminium-based catalysts were designed, and they accomplished their crucial roles in hydroboration chemistry over the years. More efficient catalysts were synthesised by tuning the ligands appropriately. The importance of lithium in the Li/Al bimetallic catalyst system is well described. Moreover, simple commercially available $\mathrm{LiAlH}_{4}$ was also applied, using it in a catalytic amount with reduced toxicity. The relevant reaction mechanism of hydroboration of different functionalities is suitably documented in this review. This emerging field of aluminium catalysis could reduce the amount of catalyst used to as low as $0.1 \mathrm{~mol} \%$. Thus, significant application of this process on a large industrial scale would be promising. Another challenge for researchers in the near future would be the development of a heterogeneous version of aluminium catalysts. This will lead to increased catalyst reusability, which will further improve product purity and atomefficiency of the whole process. This in turn will be much praised as being environment-friendly. Further structural development of aluminium complexes in terms of designing new ligands may lead to a bench-stable yet reactive catalyst which can take part in catalysis even in the presence of impurities or in the open air. In this context, it is noteworthy to mention a recent report by Cowley, Thomas and co-workers on dehydrogenative $\mathrm{C}(\mathrm{sp})-\mathrm{H}$ borylation catalysed by Al-complex. ${ }^{38}$ In situ generation of an amine tethered aluminium catalyst (FLP) can switch the alkyne hydroalumination reaction to the $\mathrm{C}(\mathrm{sp})-\mathrm{H}$ borylation. Thus, a suitable design of the Al-catalyst is capable of changing the typical reactivity of alkynes with HBpin to produce the expected hydroboration product. In this case, the role of amine to generate intramolecular FLP type catalyst is crucial to furnish high chemo-selectivity towards the formation of $\mathrm{C}(\mathrm{sp})-\mathrm{H}$ borylation product. In general, we believe that this review on the scope of aluminium complexes as catalysts in the hydroboration reaction will provide deep insight into the properties of various aluminium complexes and their progressive development over the years. This will lead to a better understanding of their catalytic properties and mechanisms, which could help develop more reactive and selective aluminium complexes for use in hydroboration reactions.

\section{Author contributions}

S.R. and A.D. conceptualised the structure of the review. A.D. wrote the review. A.D., S.R. and T.K.P checked and revised the draft of the review. We thank Himadri Karmakar for his help in preparing the solid-state structures.

\section{Conflicts of interest}

There are no conflicts to declare.

\section{Acknowledgements}

This work was supported by the Indian Institute of Technology Hyderabad (IIT H).

\section{Notes and references}

Keywords: Hydroboration - Aluminium · carbonyl compounds . alkynes $\cdot$ alkenes $\cdot$ nitriles

A.-M. Carroll, T. P. O'Sullivan and P. J. Guiry, Adv. Synth. Catal., 2005, 347, 609-631.

C. C. Chong and R. Kinjo, ACS Catal., 2015, 5, 3238-3259.

D. Hayrapetyan and A. Y. Khalimon, Chem. Asian J., 2020, 15, 2575-2587.

4 S. Rej, A. Das and T. K. Panda, Adv. Synth. Catal., 2021, 363, 4818-4840.

5 J. W. B. Fyfe and A. J. B. Watson, Chem, 2017, 3, 31-55.

6 B. S. L. Collins, C. M. Wilson, E. L. Myers and V. K. Aggarwal, Angew. Chem. Int. Ed., 2017, 56, 11700-11733.

7 J. V. Obligacion and P. J. Chirik, Nat. Rev. Chem., 2018, 2, 1534.

8 W. Fan, L. Li and G. Zhang, J. Org. Chem., 2019, 84, 59875996.

C. Nájera, I. P. Beletskaya and M. Yus, Chem. Soc. Rev., 2019, 48, 4515-4618.

M. L. Shegavi and S. K. Bose, Catal. Sci. Technol., 2019, 9, 3307-3336.

11 K. Kuciński and G. Hreczycho, Green Chem., 2020, 22, 52105224.

A. D. Bage, K. Nicholson, T. A. Hunt, T. Langer and S. P. Thomas, ACS Catal., 2020, 10, 13479-13486.

H. C. Brown and B. C. S. Rao, J. Am. Chem. Soc., 1956, 78, 5694-5695.

H. C. Brown and G. Zweifel, J. Am. Chem. Soc., 1961, 83, 486487.

H. C. Brown, N. R. Ayyangar and G. Zweifel, J. Am. Chem. Soc., 1964, 86, 397-403.

I. Beletskaya and A. Pelter, Tetrahedron, 1997, 53, 49575026.

K. Burgess and M. J. Ohlmeyer, Chem. Rev., 1991, 91, 11791191.

S. Pereira and M. Srebnik, J. Am. Chem. Soc., 1996, 118, 909910.

M. J. Geier, C. M. Vogels, A. Decken and S. A. Westcott, J. Organomet. Chem., 2009, 694, 3154-3159.

D. Mannig and H. Noth, Angew. Chem. Int. Ed. Engl., 1985, 24, 878-879.

C. E. Tucker, J. Davidson and P. Knochel, J. Org. Chem., 1992, 57, 3482-3485.

D. J. Parks and W. E. Piers, Tetrahedron, 1998, 54, 1546915488.

D. J. Parks, W. E. Piers and G. P. A. Yap, Organometallics, 1998, 17, 5492-5503.

H. Stachowiak, J. Kazmierczak, K. Kucinski and G. Hreczycho, Green Chem., 2018, 20, 1738-1742.

A. D. Bage, T. A. Hunt and S. P. Thomas, Org. Lett., 2020, 22, 4107-4112.

N. Miyaura and A. Suzuki, Chem. Rev., 1995, 95, 2457-2483.

A. Suzuki, J. Organomet. Chem., 1999, 576, 147-168.

A. A. Thomas, A. F. Zahrt, C. P. Delaney and S. E. Denmark, J. Am. Chem. Soc., 2018, 140, 4401-4416. 

and A. J. B. Watson, J. Org. Chem., 2016, 81, 3942-3950.

Z. Yang, M. Zhong, X. Ma, S. De, C. Anusha, P. Parameswaran and H. W. Roesky, Angew. Chem. Int. Ed., 2015, 54, 1022510229. 2010, 6, DOI:10.3762/bjoc.6.6.

M. M. Heravi, V. Zadsirjan, P. Saedi and T. Momeni, RSC Adv., 2018, 8, 40061-40163.

A. Caise, D. Jones, E. L. Kolychev, J. Hicks, J. M. Goicoechea and S. Aldridge, Chem. Asian J., 2018, 24, 13624-13635.

A. Caise, E. L. Kolychev, J. Hicks, M. Á. Fuentes, J. M. 65 Goicoechea and S. Aldridge, Dalt. Trans., 2019, 48, 1084510852.

D. R. Willcox, D. M. De Rosa, J. Howley, A. Levy, A. Steven, G. S. Nichol, C. A. Morrison, M. J. Cowley and S. P. Thomas, Angew. Chem. Int. Ed., 2021, 60, 20672-20677.

W. Li, X. Ma, M. G. Walawalkar, Z. Yang and H. W. Roesky, 68 Coord. Chem. Rev., 2017, 350, 14-29.

Y. Liu, J. Li, X. Ma, Z. Yang and H. W. Roesky, Coord. Chem. 69 Rev., 2018, 374, 387-415.

R. Noyori, I. Tomino and M. Nishizawa, J. Am. Chem. Soc., 70 1979, 101, 5843-5844.

R. Noyori, I. Tomino, Y. Tanimoto and M. Nishizawa, J. Am Chem. Soc., 1984, 106, 6709-6716.

R. Noyori, I. Tomino, M. Yamada and M. Nishizawa, J. Am. Chem. Soc., 1984, 106, 6717-6725.

A. J. Blake, A. Cunningham, A. Ford, S. J. Teat and S Woodward, Chem. Eur. J., 2000, 6, 3586-3594.

K. Jakhar, M. K. Barman and S. Nembenna, Org. Lett., 2016, 18, 4710-4713.

D. Franz, L. Sirtl, A. Pöthig and S. Inoue, Z. Anorg. Allg. Chem., 2016, 642, 1245-1250.

L. E. Lemmerz, R. McLellan, N. R. Judge, A. R. Kennedy, S. A Orr, M. Uzelac, E. Hevia, S. D. Robertson, J. Okuda and R. E. Mulvey, Chem. Eur. J., 2018, 24, 9940-9948.

V. A. Pollard, S. A. Orr, R. McLellan, A. R. Kennedy, E. Hevia and R. E. Mulvey, Chem. Commun., 2018, 54, 1233-1236.

V. A. Pollard, M. Á. Fuentes, A. R. Kennedy, R. McLellan and R. E. Mulvey, Angew. Chem. Int. Ed., 2018, 57, 10651-10655. T. Peddarao, N. Sarkar and S. Nembenna, Inorg. Chem., 2020, 59, 4693-4702.

B. Prashanth, M. Bhandari, S. Ravi, K. R. Shamasundar and S. Singh, Chem. Eur. J., 2018, 24, 4794-4799.

D. Jin, X. Ma, Y. Liu, J. Peng and Z. Yang, Appl. Organomet. Chem., 2019, 33, 1-10.

3 G. Zhang, J. Wu, H. Zeng, M. C. Neary, M. Devany, S. Zheng and P. A. Dub, ACS Catal., 2019, 9, 874-884.

A. Suzuki, Heterocycles, 2010, 80, 15-43.

A. Suzuki, Angew. Chem. Int. Ed., 2011, 50, 6722-6737.

I. P. Beletskaya, F. Alonso and V. Tyurin, Coord. Chem. Rev., 2019, 385, 137-173.
Z. Yang, M. Zhong, X. Ma, K. Nijesh, S. De, P. Parameswaran and H. W. Roesky, J. Am. Chem. Soc., 2016, 138, 2548-2551. A. Bismuto, S. P. Thomas and M. J. Cowley, Angew. Chem. Int. Ed., 2016, 55, 15356-15359.

A. Bismuto, M. J. Cowley and S. P. Thomas, ACS Catal., 2018, 8, 2001-2005.

F. Li, X. Bai, Y. Cai, H. Li, S.-Q. Zhang, F.-H. Liu, X. Hong, Y. Xu and S.-L. Shi, Org. Process Res. Dev., 2019, 23, 1703-1708.

A. Harinath, I. Banerjee, J. Bhattacharjee and T. K. Panda, New J. Chem., 2019, 43, 10531-10536.

A. K. Jaladi, W. K. Shin and D. K. An, RSC Adv., 2019, 9, 26483-26486.

A. K. Jaladi, H. Kim, J. H. Lee, W. K. Shin, H. Hwang and D. K. An, New J. Chem., 2019, 43, 16524-16529.

N. Sarkar, S. Bera and S. Nembenna, J. Org. Chem., 2020, 85, 4999-5009.

K. Hobson, C. J. Carmalt and C. Bakewell, Inorg. Chem., 2021, 60, 10958-10969.

A. Harinath, J. Bhattacharjee and T. K. Panda, Adv. Synth. Catal., 2019, 361, 850-857.

W. Liu, Y. Ding, D. Jin, Q. Shen, B. Yan, X. Ma and Z. Yang, Green Chem., 2019, 21, 3812-3815.

Y. Ding, X. Ma, Y. Liu, W. Liu, Z. Yang and H. W. Roesky, Organometallics, 2019, 38, 3092-3097.

Q. Shen, X. Ma, W. Li, W. Liu, Y. Ding, Z. Yang and H. W. Roesky, Chem. Eur. J., 2019, 25, 11918-11923.

C. Chia, Y. Teo, N. Cham, S. Y. Ho, Z. Ng, H. Toh, N. Mézailles and C. So, Inorg. Chem., 2021, 60, 4569-4577. 